\title{
Flora da Reserva Ducke, Amazonas, Brasil: Flacourtiaceae
}

Sue Zmarzty ${ }^{1}$

Flacourtiaceae DC., Prodr. 1: 255. 1824.

Alford, M. H. 2005a. Systematic studies in Flacourtiaceae. Dissertation. Cornell University, Ithaca, 290p.

Alford, M. H. 2005b. Nomenclatural Innovations in Neotropical Salicaceae: Novon 16(3): 293-298.

Alford, M. H. 2007. Samydaceae. Version 06 February 2007 (under construction). http://tolweb.org/Samydaceae/ 68361/2007.02.06 in The Tree of Life Web Project: http://tolweb.org/

Angiosperm Phylogeny Group. 2003. An update of the Angiosperm Phylogeny Group Classification for the orders and families of flowering plants: APG II. Bot. Journ. Linn. Soc. 141(4): 399-436.

Chase, M. W.; Zmarzty, S.; Lledó, M. D.; Wurdack, K. J.; Swensen, S. M. \& Fay, M. F. 2002. When in doubt, put it in Flacourtiaceae: a molecular phylogenetic analysis based on plastid $r b c L$ DNA sequences. Kew Bull. 57: 141-181.

Maas, P. J. M. \& Westra, L. Y. Th. 1993. Neotropical Plant Families. Koeltz Scientific Books, Germany/USA.

Sleumer, H. O. 1980. Flacourtiaceae. Flora Neotropica Monograph 22. New York Botanical Garden, New York. Pp. 1-499.

Stevens, P. F. 2001 (onwards). Angiosperm Phylogeny Website. Version 7, May 2006 [and more or less continuously updated since]. http://www.mobot.org/MOBOT/research/APweb

Trees or shrubs, branches unarmed or spiny; hairs usually simple, rarely stellate; stipules often caducous. Leaves alternate, rarely opposite, penninerved or 3-5-nerved from base, often pellucid-punctate/lineate, leaf margin entire or toothed, teeth sometimes glandular. Inflorescences terminal, axillary or supra-axillary, flowers solitary or in fascicles, glomerules, cymes, racemes or panicles; bracts generally small, sometimes with several together forming a cushion; pedicels often articulate. Flowers bisexual or unisexual, actinomorphic; sepals (2-)3-5(-6), rarely more, usually imbricate or valvate, free or connate in basal part, sometimes petaloid and conspicuous; petals 3-10, free, mostly alternating with sepals, or petals absent; floral disc often present, intra-, extra-, or interstaminal, variable in form: rudimentary or well-developed, entire, lobed, of free glands, scale-like, or cup-like with stamens inserted on rim, the stamens alternating with disc lobes; stamens 5-many, hypogynous or perigynous, filaments free, usually slender; anthers versatile, dorsi- or basifixed, 2-locular, globose to linear, generally opening by longitudinal slits; connective sometimes glandular, sometimes extending beyond locules; ovary superior (in Ducke genera) or rarely semi-inferior, 1-locular (in Ducke genera) or falsely plurilocular; placentas 3-several, parietal, pluri-ovulate; styles 1-10 (rarely $-40 ; 1-10$ in Ducke species) simple or branched at apex, stigma(s) capitate to flattened-lacerate. Fruit baccate or capsular (Ducke material), rarely a drupe or samara, dry or fleshy, indehiscent or dehiscing by (2-)3-5(-7) valves, sometimes resinous; seed 1-many, often compressed, sarcotesta and/or aril often present.

Approximately 87 genera with 900 species. Trees and shrubs, in evergreen rainforest, semi-deciduous transitional forest and wooded savanna, in tropical and subtropical regions, pantropical. At low to high altitude, in S. America occasionally above the Andean tree-line (Pineda). Five genera from the Reserva Ducke: Carpotroche, Casearia, Laetia, Ryania, Xylosma, with a further 2 likely: Lindackeria, Neoptychocarpus (Hopkins 2007, pers. comm.).

Descriptions given here are based mostly on material from the Reserva Ducke (measurements etc. taken from dried material) but also draw from Sleumer (1980). For two species likely within the Reserve but not yet

${ }^{1}$ Royal Botanic Gardens, Kew, Richmond, Surrey TW9 3AB, U.K. s.zmarzty@ rbgkew.org.uk 
found, Neoptychocarpus apodanthus (Kuhlm.) Buchheim and Lindackeria paludosa (Benth.) Gilg, brief descriptions are given for convenience, drawn mostly from Sleumer (1980). Lacistema and Lozania, also in Amazonas, are considered a separate family, Lacistemataceae.

Morphologically Flacourtiaceae are extremely heterogeneous in floral and fruit morphology, making recognition at family level difficult. The best family characteristics (1locular ovary, parietal placentation) are difficult to observe in the field, and even these are shared by other families (e.g. Violaceae). Identification is easier at generic level, although several genera (e.g. Xylosma) can be confused with Euphorbiaceae. Flacourtiaceous flowers are often very small and not easily observed without magnification and dissection.

Chase et al. (2002) transferred many flacourt genera, including Flacourtia itself, to Salicaceae, expanding the latter into a temperatetropical family of $c a$. 50 woody genera. The remaining 30 flacourt genera were transferred to the hitherto small South African family, Achariaceae. The new circumscriptions were based on molecular analyses, comparative morphology, wood anatomy, palynology and phytochemistry, and although not universally accepted, are currently supported by the Angiosperm Phylogeny Group (APG 2003, Stevens 2001 onwards). Some of the genera transferred by Chase et al. to the Salicaceae are by Alford (2005a, 2005b, 2007) housed instead in a separate family, the Samydaceae. Terminology: Leaf punctation/striation, the occurrence of small translucent dots and lines in the leaf lamina, is best observed at x $10 \mathrm{mag}$. (magnification), from the upper surface with the leaf held up to the light. For secondary venation a range is given for the angle at which the secondary veins branch from the midvein; this range refers to the variation of angles within a single leaf, with the narrower angles occurring towards the leaf base. The number of secondary veins includes the weak extreme basal pair(s). Pericarp thickness was measured from rehydrated dried material.

\section{Key to genera of Flacourtiaceae in Reserva Ducke: material in flower}

1. Petals 6-10; sepals 3.

2. Petals $1.5-3 \mathrm{~cm}$ long; styles 5-7; petioles $0.5-1.5 \mathrm{~cm}$ long ................... 1. Carpotroche

2. Petals $0.6-0.8 \mathrm{~cm}$ long; style 1 ; petioles $2-8 \mathrm{~cm}$ long ............................ 7. Lindackeria

1. Petals absent; sepals 4-5.

3. Calyx $2-4 \mathrm{~cm}$ long, petaloid, outside densely stellate-hairy ....

2. Ryania

3. Calyx $<1 \mathrm{~cm}$ long, petaloid or not, outside not stellate-hairy.

4. Calyx divided practically to base into separate sepals, calyx tube absent; disc comprised of minute to small globose to squamular lobes in an extrastaminal row, or obsolete and barely discernable around ovary base.

5. Flowers bisexual; sepals strongly reflexed at anthesis; style long $(5-6 \mathrm{~mm})$ or anthers long (1.4-2 mm), anthers linear to narrowly triangular; disc obsolete, barely discernable around ovary base

5. Flowers usually unisexual; sepals erect to spreading at anthesis; style (plus stigma) short $(0.5-0.8 \mathrm{~mm})$ or anthers minute (ca. $0.2 \mathrm{~mm}$ long); disc comprised of an extrastaminal row of more or less globose to squamular connate lobes (staminate and bisexual flowers) or an extragynoecial annulus (carpellate flowers), disc clearly visible at $\times 10-20$ mag. (at least in floral dissection) 4. Xylosma

4. Calyx connate in lower part to form a short to long calyx tube, or disc lobes linear, narrow-oblong or narrow-clavate, usually hairy, alternating with stamen filaments or set in an intrastaminal row, $1 / 4-3 / 4$ as long as stamen filaments. 
6. Flowers bisexual; disc with linear to narrow-oblong or narrow-clavate usually hairy lobes $1 / 4$ $3 / 4$ as long as stamen filaments, these alternating with stamen filaments or set in an intrastaminal row; filaments of longest stamens linear-cylindrical

5. Casearia*

6. Flowers unisexual; disc lobes absent, all stamen filaments broadly triangular, each with a pair of small lateral lobes 6. Neoptychocarpus*

*Without floral dissections $C$. resinifera can easily be confused with Neoptychocarpus apodanthus. Both species have long narrowly oblong leaves and small flowers in axillary clusters. Leaf venation is a useful distinguishing character. In $C$. resinifera the secondary veins branch from the midvein at $(60-) 70-80^{\circ}$. Approaching the leaf margin they curve upward towards the leaf apex, run alongside the leaf margin for a short distance and finally become much finer and less prominent before joining the secondary vein above in an inconspicuous manner. In N. apodanthus the secondary veins are almost perpendicular to the midvein, branching from it at almost $90^{\circ}$. On approach to the leaf margin they fork widely into two branches of more or less equal strength, one branch turning towards the leaf apex and the other towards the leaf base; the branches of adjacent secondary veins form conspicuous loops at the leaf margin. C. resinifera is often resinous at the terminal bud and inflorescence bases, $N$. apodanthus is not resinous.

\section{Key to genera of Flacourtiaceae in Reserva Ducke: material in fruit}

1. Pericarp covered with broad papery crests, smallish conical more or less apiculate protuberances, short conical bristles, short flattened-triangular emergences or thin low irregular lamellae up to $2 \mathrm{~mm}$ high.

2. Pericarp covered with crowded papery crests up to $2.5 \mathrm{~cm}$ tall/broad, capsule including crests $5.5-10 \mathrm{~cm}$ diameter 1. Carpotroche

2. Fruit without tall/broad papery crests, diameter various.

3. Pericarp covered with smallish conical more or less apiculate protuberances, or with short conical bristles; persistent style elongate; petioles $2-8 \mathrm{~cm}$ long; leaf secondary veins branching from midvein at $45-60^{\circ}$ 7. Lindackeria

3. Pericarp covered with smallish flattened triangular emergences or thin low irregular lamellae up to $2 \mathrm{~mm}$ high; style if persistent very short; petioles $0.3-0.6 \mathrm{~cm}$ long; leaf secondary veins branching from midvein at $80-90^{\circ}$ 6. Neoptychocarpus

1. Pericarp smooth, wrinkled or veined, sometimes with fine more or less straight longitudinal grooves or ridges marking the valve margins.

4. Pericarp outside densely stellate-hairy, the indumentum reddish- or yellowish-brown, drying yellowish-, greyish- or reddish brown 2. Ryania

4. Pericarp outside not densely stellate-hairy.

5. Bract subtending each pedicel conspicuous, 5-6 mm long, with basal part up to $3 \mathrm{~mm}$ long, narrowly tubular, closely sheathing pedicel base, apical part 2-4 mm long, cupshaped 3. Laetia (L. cupulata)

5. Bract subtending each pedicel not as above.

6. Fruit baccate, with small annular disc persistent at base (at least until fruit abscission) and short stout style and flattened-deltoid, semi-lunate to u-shaped stigmas persistent at apex; leaf lamina not pellucid-punctate/striate; serrations of leaf margin each with an apical circular gland on underside 4. Xylosma

6. Fruit baccate or capsular, annular disc absent, style/stigmas of various form persistent or not; leaf lamina usually pellucid-punctate/striate; serrations of leaf margin each without an apical circular gland on underside.

7. Pedicel in mature fruit $10-15 \mathrm{~mm}$ long, capsule $10-20 \mathrm{~mm}$ long, globose to broadly trigonal-globose when mature (although immature fruit narrowly ellipsoid), outside glabrous, with three distinct longitudinal bands of transverse wrinkles, capsule valves broadly elliptic to subcircular, rounded at apex; seed 30-40, held together as a mass; reflexed sepals often persistent 3. Laetia (L. procera)

7. Pedicels and mature fruit not as above 5. Casearia 


\section{Carpotroche}

Carpotroche Endl., Gen. Pl. 918. 1839.

Shrubs or small trees; branchlets unarmed.

Stipules caducous. Leaves alternate, often crowded at twig tips, petiolate, penninerved. Inflorescences axillary, cymose in foliate or defoliate axils, or from old wood, 1-fewflowered. Flowers unisexual or rarely bisexual (plants dioecious, polygamous-monoecious or polygamous-dioecious); sepals (2-)3, imbricate, free; petals (4-)6-9(-12), imbricate, white or cream, papery; disc absent. Staminate flowers: stamens many, (10-) 35-80, free, crowded on receptacle, filaments short relative to anthers, anthers linear, basifixed; rudiment of ovary 0 . Carpellate flowers: ovary smooth or with 4 or more ridges or crests; styles 4-8(10), short, connate at base, stigmas capitate or shortly lacerate-palmatifid; stamens and staminodes 0 . Capsule mostly 3-7 cm diam. (larger in $C$. brasiliensis), generally ellipsoid or globose, with 4 or more ridges or crests, dehiscent by $3-5(-7)$ valves, pericarp generally thick, often fibrous. Seed usually many, 8-20 mm long, angular by compression, immersed in pulp, occasionally reported to be sarcotestal or arillate.

Eleven species in Central America and Amazonian S. America. One species from the Reserva Ducke. Also from Brazilian Amazonas: C. amazonica, C. froesiana, C. grandiflora, C. integrifolia, C. longifolia (Sleumer 1980).

1.1 Carpotroche crispidentata Ducke, Arch. Inst. Biol. Veg. 4: 55. 1938.

Fig. 1

Tree to $10 \mathrm{~m}$ tall. Terminal bud softly and densely sericeous, hairs cream to greyishbrown. Twig tips and young branchlets softly and densely pilose, hairs adpressed to spreading, pale yellowish- or greyish-brown, older branchlets becoming glabrous. Lenticels on young growth sparse, concealed by indumentum, on older branchlets conspicuous, sometimes numerous. Stipules 8-12 mm long, lanceolate to linear, acute at apex, softly and densely sericeous outside, sparsely so inside, caducous. Leaves usually 3-6-crowded at twig tips; petioles 5$11 \times 1.5-2 \mathrm{~mm}$, grooved above, persistently dense-pilose throughout; blades (14.5-)18-25 $\times 6.5-11.5(-12.5) \mathrm{cm}$ long, 2-2.7 times as long as broad, obovate; base narrowly to broadly acute, cuneate, extreme base acute, truncate or rounded; apex cuspidate-acuminate, acumen $2.5-3.5 \mathrm{~cm}$ long, extreme tip acute, minutely mucronate; lamina thin-chartaceous, pilose on both surfaces including venation, more densely so beneath. Midvein raised and conspicuous below. Secondary veins (8-)10-14 pairs, curved-ascending, branching from midvein at (50-)60-80 $0^{\circ}$, above fine, impressed and rather obscure, below raised and conspicuous; midvein and secondary veins densely pilose, especially below, indumentum pale-tawny, soft. Leaf margin shallowly dentate to crenate-undulate, or almost entire. Infloresences in foliate axils, cymose, 1-3-flowered, 2-4 cm long (excluding flower), raches pilose. Bracts 2-3, narrowly lanceolate, 7-10 $\mathrm{mm}$ long, pilose to sericeous outside, inside glabrous except for sericeous base, similar to stipules. Pedicel in flower $c a$. (8-) $10 \mathrm{~mm}$ long, articulate at base, densely pilose especially above articulation. Bud globose. Flowers unisexual or rarely also bisexual on the same plant, sweetly scented. Staminate flowers: sepals 3, spreading at anthesis, 15$18 \mathrm{~mm}$ long, elliptic-suborbicular, hooded, outside densely sericeous in areas exposed in bud, glabrous elsewhere, greenish-white when fresh, becoming reddish, inside glabrous; petals $7-10$, spreading at anthesis, $20-35 \times 8-20 \mathrm{~mm}$, variable in size within one flower (at least when dried), obovate to spatulate-obovate, with rounded apex, white, sericeous throughout on both sides, indumentum drying cream-coloured, greyish or yellowish; stamens many (ca.40), outermost filaments 3-6 mm long, innermost filaments 2-3(-4) $\mathrm{mm}$ long, all filaments sericeous throughout; anthers 6-8 mm long, linear, sericeous, glabrous towards apex, connective linear, not swollen, sericeous except towards glabrous apex, apex projecting by $0.2-$ $0.5 \mathrm{~mm}$, tip bilobed; rudiment of ovary 0 . Carpellate flowers: solitary, sepals and petals as in staminate flower, ovary subglobose, sericeous to tomentulose, with 10-12 vertical, membranous, crenate wings; styles 5(-7), sericeous to tomentose; stigmas short-lacerate 


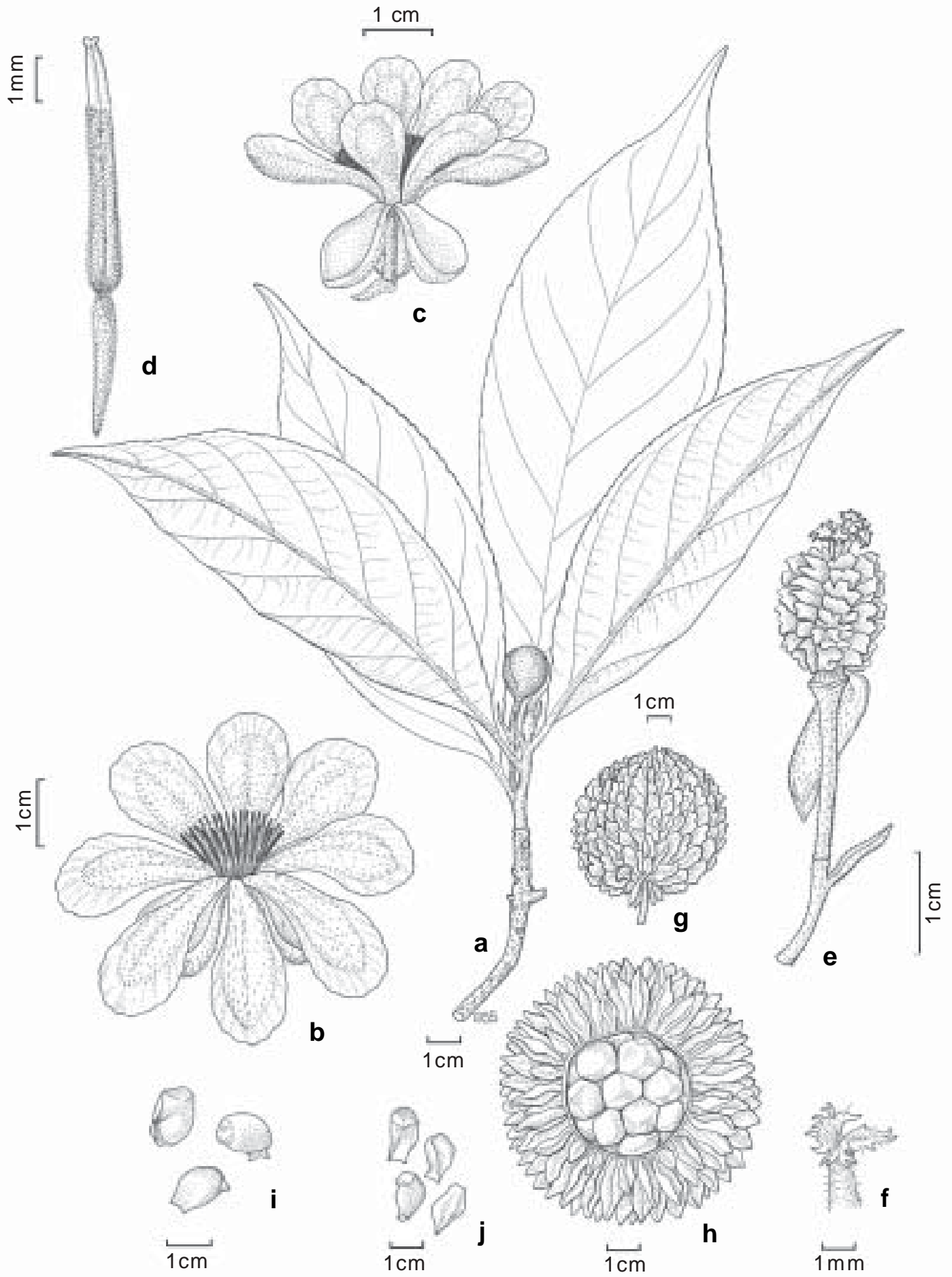

Figure 1 - Carpotroche crispidentata - a. habit; b-c. flower (male); d stamen; e. young fruit; f. style; g. fruit; h. fruit, crosssection; i-j. seed. (a. composite illustration from Sothers 627 (shoot apex) and Assunção 289 (bud); b-d. from Sothers 627; e. composite illustration from Granville 7882 and Sothers 669; f. from Sothers 669; g. from Rodrigues \& Lima 2426; h, j. from Sothers 731; i. from Albuquerque s.n. (INPA 5354)). Drawn by Susanna Stuart-Smith. 
(Sleumer 1980). Pedicel in fruit ca. $20 \mathrm{~mm}$ long, sericeous. Capsule globose, $5.5-10 \mathrm{~cm}$ diameter, yellow or yellowish green when fresh, drying brown, pericarp $c a .1 .5 \mathrm{~cm}$ thick, outside with many densely set longitudinal crests, these (1-)2-2.5 cm tall, erect, papery, pleated, pubescent; pericarp inside glabrous, pale brown. Calyx remnants sometimes persistent at capsule base. Seed 30-40, immersed in pulp, 13-15 × ca. $8 \mathrm{~mm}$, compressed obovoid to polygonal, whitish, drying dark brown, sparsely puberulent to glabrous, minutely and shallowly tuberculate; aril entire, adnate to base and one side of seed, reddish when fresh, marzipan-scented.

Carpellate flowers (not seen from the Reserva Ducke) described from Sleumer (1980).

Habitat within Reserva Ducke: understorey tree of lowland forest, often near streams; on sandy or clay soil.

Distribution within Reserva Ducke: "floresta de baixio", "floresta de platô".

General distribution: Venezuela, Brazil, French Guiana.

Material from the Reserva Ducke: Albuquerque s.n. (INPA 5354); Assunção 289 (INPA 186886); Coêlho s.n. (INPA 5200), s.n. (INPA 6541); Ferreira s.n.(INPA 4190); Loureiro s.n. (INPA 16579); Ramos 1858; Rodrigues 2168, 5518, 6885; Rodrigues and Coêlho 5621, 7317; Rodrigues \& Lima 2426; Rodrigues \& Loureiro 5759; Sothers 627, 669, 731; Vicentini 701. Carpotroche cf. crispidentata: Ramos 1858, Vicentini et al. 701.

Additional material: BRAZIL. AMAZONAS: Borba, rio Madeira, 23.VI.83 (fr) Cid 3901 (INPA K NY). FRENCH GUIANA: Montagne Bellevue de l'Inini, 27.VIII.1985 (young fr) Granville et al. 7882 (CAY K P U INPA).

\section{Ryania}

Ryania Vahl, Eclog. Amer. 1: 51. 1796.

Monachino, Lloydia 12: 1-29. 1949.

Shrubs or small trees; branchlets unarmed. Stipules often caducous; hairs simple and/or stellate. Leaves alternate, distichous, petiolate, penninerved, impunctate. Inflorescences axillary, fasciculate or with very much abbreviated rachis, 1-few-flowered. Flowers bisexual; sepals 5 , connate at base into a very short tube, imbricate, petaloid, spreading or erect; petals 0 ; stamens many (30-70), free except at connate base, inserted in 2-3 series at apex of calyx tube, filaments filiform, anthers oblong to linear, slightly sagittate, dorsifixed near base; disc erect, coroniform, with upper half to two-thirds mostly of double thickness, as if inwardly downfolded, outside longitudinally ridged; ovary 1-locular, placentas 3-9, multi-ovulate; style entire or 3-9-fid at apex. Capsule indehiscent to tardily dehiscent, globose to pyriform, $1-6 \mathrm{~cm}$ long, suberose or with spongy tuberculate emergences, subhirsute to stellate-tomentose, with perianth remnants sometimes persistent at base. Seed many, arillate.

Eight species in Central and South America, from Nicaragua to Peru and Brazil. Two species from the Reserva Ducke. Also from Brazilian Amazonas: $R$. angustifolia, $R$. mansoana $R$. speciosa var. bicolor, var. minor, var. tomentella, var. tomentosa, $R$. spruceana (Sleumer 1980).

Probably all species of Ryania are extremely poisonous, all parts of the plant being highly toxic to both cold- and warm-blooded animals. Derivatives are used as animal poisons and insecticides (Sleumer 1980).

\section{Key to species of Ryania in Reserva Ducke}

1. Twig tip indumentum not tufted*; leaves $10-18 \times 2.5-5.5 \mathrm{~cm}, 2.5-5$ times as long as broad; sepals 20-30 $\times 2-6 \mathrm{~mm}$; disc ca $2 \mathrm{~mm}$ tall, erect hairs on disc rim almost as long as height of disc; capsule reddish, drying greyish- to chestnut-brown, indumentum not especially tufted ...

1. R. speciosa var. subuliflora

1. Twig tip indumentum tufted; leaves (16-)18-24 $\times(5-) 6-8.5 \mathrm{~cm}, 2.3-3.7$ times as long as broad; sepals $25-40 \times 6-16 \mathrm{~mm}$; disc $3-5 \mathrm{~mm}$ tall, erect hairs on rim about $1 / 3 \mathrm{x}$ height of disc or less; capsule greyish- or greenish-yellow, indumentum tufted 2. R. pyrifera

\footnotetext{
*A 'tufted' indumentum is here defined as a dense indumentum in which some hairs or tufts of hairs stand taller than others, giving the indumentum an almost granular appearance at magnifications up to $c a$. $x 10$ mag. The term 'tufted' used in this sense should not be confused with the appearance of a sparse stellate indumentum where each separate stellate hair resembles a small tuft against a glabrous background.
} 
2.1 Ryania speciosa Vahl, Eclog. Amer. 1: 51, t.9. 1796.

A species in which 10 varieties are recognised, some of which intergrade (Sleumer (1980) after Monachino (1949)); one variety recorded for the Reserva Ducke.

2.1a Ryania speciosa var. subuliflora (Sandw.) Monach., Lloydia 12: 14. 1949.

Ryania pyrifera var. subuliflora Sandwith, Jour. Arn. Arb. 24: 219. 1943.

Shrub or small tree to $6 \mathrm{~m}$ tall; terminal bud densely stellate-tomentose, pale yellowishbrown or tawny-brown;young twigs and branchlets sulcate/ridged, indumentum persistent, dense, finely pale- or tawny-brown tomentose, not tufted; older twigs and branchlets eventually glabrous, pale-greyish or with pale greyish-brown patches; lenticels inconspicuous; stipules 4-6 mm long, subulate, expanded at base, with acute apex, densely stellate-tomentose on both surfaces, subpersistent to caducous; petioles $2-5 \times 1-1.5 \mathrm{~mm}$, stellate-hairy at least when young. Leaves $10-18 \times 2.5-5.5 \mathrm{~cm}, 2.5-5$ times as long as broad, chartaceous to firmchartaceous, oblong to long-oblong; base acute, slightly oblique; apex cuspidate-acuminate, acumen $0.7-1.3 \mathrm{~cm}$ long, extreme tip obtuse to rounded; lamina upper surface glabrous except for dense, adpressed to semi-spreading, stellate, pale- to tawny-brown indumentum on midvein, lower surface usually sparsely stellatehairy throughout, slightly scabrid and rough to touch; midvein raised below, conspicuous; secondary veins $6-10$ pairs, curved-ascending, branching from midvein at $40-70^{\circ}$, raised and conspicuous below; leaf margin entire, slightly undulate. Inflorescences in foliate (usually) or defoliate axils of young twigs; bracts coriaceous, densely stellate-hairy outside throughout, also inside towards apex, variable in length and shape, $1.5-5 \mathrm{~mm}$ long, subulate, cuspidate or caudate, keeled, base expanded, apex acute; bud longconical, base rounded, apex acute; pedicel in flower 3-7 mm long, articulate towards base, densely ferruginous stellate-hairy, indumentum tufted. Flower sweetly aromatic; sepals 5, 20$30 \times 2-6 \mathrm{~mm}$, spreading at anthesis, pale green or pinkish when fresh, narrowly oblong, apex acute, outside very densely ferruginous stellatetomentose except finely tomentulose where covered in bud, inside finely and densely whitish-tomentulose, indumentum pale greyishbrown when dried; stamen filaments $2-3 \mathrm{~cm}$ long, filamentous, glabrous except for a few long hairs at base; anther 7-8 mm long, linear, glabrous, connective projecting for $c a .0 .5 \mathrm{~mm}$, acute at apex, glabrous; disc ca. $2 \mathrm{~mm}$ tall, adnate to calyx at base, outside glabrous or with a few long, straight hairs towards rim, disc rim densely villous with the hairs almost as long as the disc itself, inside villous around ovary base, short-sericeous just below rim, otherwise glabrous; ovary ca $4 \mathrm{~mm}$ long, ovoid, sessile, densely sericeous-pilose, cream-rose, pale yellowish-brown when dried; placentas 4-5, slightly intrusive; style to $2.5 \mathrm{~cm}$ long, glabrous except for sericeous-pilose base, reddish, apex with 5 short recurved stigmatic branches; stigmas capitate, glabrous; pedicel in fruit $c a$. $10 \mathrm{~mm}$ long. Capsule $c a .3 .5 \mathrm{~cm}$ long, indehiscent or tardily dehiscent, globose to pyriform, reddish to reddish-brown when fresh, wrinkled and greyish- to chestnut-brown when dried; base contracting into a short stalk; apex rounded, with or without persistent style remnant; pericarp 2-2.5 mm thick, coriaceous, indumentum dense, ferruginous stellate-hairy with a few longer, straighter, paler hairs interspersed, indumentum not especially tufted, pericarp inside densely and finely adpressed ferruginous hairy between placentas, placental areas glabrous, brown, glossy. Seed 6-7 mm long, broad-obovoid to compressed-polygonal, laxly pubescent, minutely foveolate (just visible at $\times 10$ mag.), reddish-brown with darker streaks; aril dark reddish-brown.

Habitat within the Reserva Ducke: Subcanopy/understorey tree, on slopes; on clay or sandy soil.

Distribution within Reserva Ducke: "floresta de platô", floresta de vertente.

General distribution: South America from Venezuela southwards to Amazonian Brazil.

Material from the Reserva Ducke: Assunção 265; Ribeiro 872, 1146; Rodrigues 9534 (not seen); Santos 941; Sothers 501, 606; Souza 72; Vicentini 643. 
2.2 Ryania pyrifera (Rich.) Uitten \& Sleumer in Pulle, Fl. Suriname 3: 286. 1935, excl. Ryania speciosa Vahl pro syn. \& descr.).

Fig. 2

Ryania sagotiana Eichler in Mart., Fl. bras. 13(1): 491. 1871.

Small tree to $6 \mathrm{~m}$ tall, trunk chestnutgreyish-rose; terminal bud densely gold-brown stellate-tomentose; twigs and branchlets densely and finely pale-brown stellatetomentose, indumentum of youngest twig tips tufted. Older branchlets becoming glabrous, sometimes pale-greyish or with pale greyishbrown patches. Lenticels inconspicuous. Stipules 8-10×1.5-2 mm, subulate, with dense short-stellate, pale-brown hairs outside, pilose inside, caducous. Petioles $2-4 \times 2-2.5 \mathrm{~mm}$, stellate-hairy. Leaves (16-)18-24 × (5-) 6$8.5 \mathrm{~cm}, 2.3-3.7$ times as long as broad, firmchartaceous, oblong, finely and densely pellucid-punctate; base broadly acute, obtuse or subrounded, slightly oblique; apex cuspidateacuminate, acumen 0.6-1.5(-2) cm long, extreme tip acute to obtuse; lamina glabrous above except for dense, erect-stellate, rather long, gold-brown hairs on midvein, lower surface sparsely stellate-hairy throughout, the hairs short, spreading, pale-brown, slightly scabrid and rough to touch. Midvein raised below, conspicuous. Secondary veins (9-)1114 pairs, curved-ascending, branching from midvein at $60-70^{\circ}$, raised and conspicuous below. Leaf margin entire, slightly undulate. Inflorescences at anthesis in foliate or defoliate axils of young twigs and branchlets. Bracts coriaceous, densely stellate-hairy outside, glabrous inside, lowermost bracts oblong and up to $3 \mathrm{~mm}$ long, bracts around pedicel bases ovate, to $2 \mathrm{~mm}$ long. Pedicel $7-$ $12 \mathrm{~mm}$ long, articulate near base, densely tufted stellate-hairy, hairs orange-brown or pale yellow-brown. Bud ovoid to oblong-ovoid, base rounded, apex obtuse. Flower strongly and sweetly scented. Sepals $5,25-40 \times 6-16 \mathrm{~mm}$, spreading at anthesis, whitish with red basal part when fresh, oblong to lanceolate, apex obtuse, outside very densely tufted stellatehairy, hairs gold-brown to ferruginous, inside finely and densely tomentulose, indumentum pale greyish-brown when dried. Stamen filaments $2-3 \mathrm{~cm}$ long, filamentous, pubescent at base, otherwise glabrous; anther 6-7 mm long, linear, glabrous, connective projecting for ca. $0.5 \mathrm{~mm}$, acute at apex, glabrous. Disc 3-5 mm tall, adnate to calyx at base, outside rose-coloured, sericeous along ridges, hairs gold-brown to ferruginous when dried, disc rim villous, hairs about $1 / 3 \times$ length of disc or less, inside glabrous in basal part, densely gold-brown to ferruginous sericeous in upper part. Ovary 3-4 mm long, globose-ovoid, stipitate, exserted beyond disc rim, stalk to $4 \mathrm{~mm}$ long, stout, ovary and stalk densely yellow-sericeous, gold-brown when dried, placentas 6-7. Style 3-4 mm long, extending in fruit, rose-coloured, with 7 very short stigmatic branches at apex, stigmas flatcapitate. Pedicel in fruit $c a .10 \mathrm{~mm}$ long, densely stellate-hairy, hairs yellow or orangebrown. Capsule $c a .4 \mathrm{~cm}$ long, indehiscent or tardily dehiscent, globose to pyriform, greenish to reddish when fresh, drying grey-, yellowor orange-brown; base contracting into a short stalk; apex rounded, style remnant persistent; pericarp 2-2.5 $\mathrm{mm}$ thick, coriaceous, outside with dense, tufted, stellate-hairy indumentum, inside densely ferruginous-stellate hairy between placentas, placental areas glabrous, brown, glossy. Seed 6-7 mm long, broadobovoid to compressed-polygonal, laxly pubescent, minutely foveolate (pits just visible at $\times 10$ mag.), reddish brown with darker streaks; aril dark reddish-brown.

Habitat within Reserva Ducke: Forest understorey, on slopes; soil clay or clay-sand. Distribution within Reserva Ducke: "floresta de platô", "floresta de vertente".

General distribution: Guianas and Amazonian Brazil.

Material from the Reserva Ducke: Coêlho D-66; Ferreira 158 (not seen); Prance 2123 (not seen); Rodrigues 1293 (not seen), 5441 (not seen), 5983 (not seen); Sothers 152, 458, 776, 790, 948; de Souza 61 (not seen); Vicentini 451.

Additional material: BRAZIL. AMAZONAS: ManausItacoatiara Highway, km. 8, Colonia Santa Antonio, 8.IX.1966 (fl) Prance 2231 (IAN INPA K NY). 


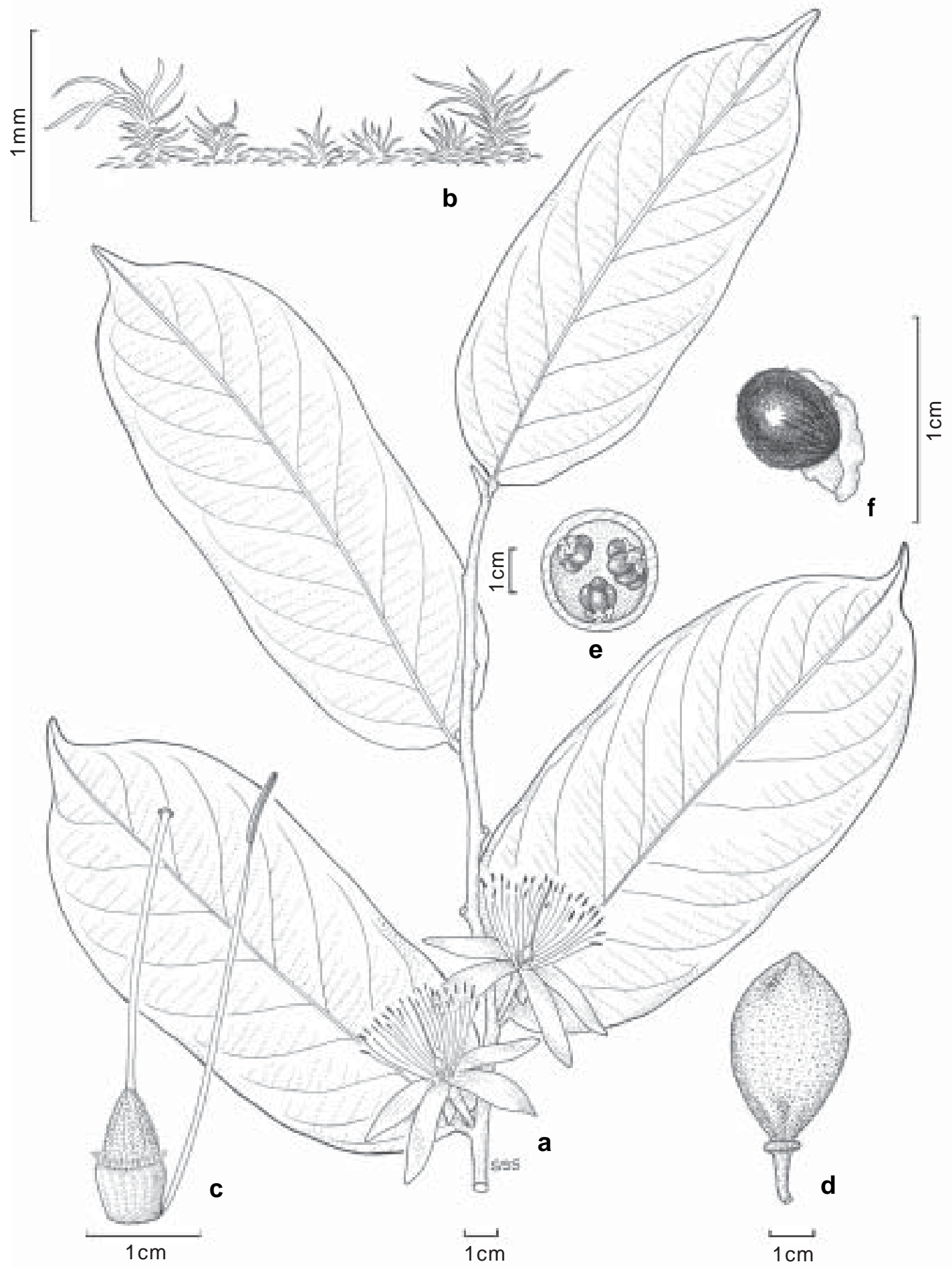

Figure 2 - Ryania pyrifera - a. habit; b. tufted indumentum; c. flower: dissected, with sepals and most of stamens removed to show single stamen, disc, ovary and style; d. fruit, entire; e. fruit, cross-section; f. seed with aril. (a. from Sothers 458; b. from Sothers 948; c. from Prance 2231; d-f. from Sothers 776). Drawn by Susanna Stuart-Smith. 


\section{Laetia}

Laetia Loefl. ex L., Syst. Nat. (ed. 10) 2: 1074. 1759.

Shrubs or trees. Branchlets unarmed. Stipules usually early caducous. Leaves alternate, distichous, petiolate, penninerved, pellucid-punctate/lineate or impunctate. Inflorescences axillary, supra-axillary, or subterminal, fasciculate or with very much abbreviated raches, few- to many-flowered, in or just above foliate or defoliate axils of young twigs. Bracts mostly small, free, rarely fused and cup-like (L. cupulata). Pedicels articulate. Flowers small, bisexual. Sepals 4-5, imbricate, connate at base, almost petaloid, pellucidpunctate-lineate, reflexed at anthesis. Petals 0. Disc obsolete, barely discernable around ovary base, adnate to calyx. Stamens 10-80, inserted on disc, uniseriate or in 2-3 rows, filaments free, slender; anthers small, dorsi- or basifixed. Ovary 1-locular; placentas 3-6, multi-ovulate. Style simple or 3-branched at apex, stigma(s) capitate. Capsule berry-like, dehiscent by 3 valves, pericarp coriaceous. Seed 1-ca. 40, arillate.

Ten species in Central and South America; two species from the Reserva Ducke. Also from Brazilian Amazonas: L. coriacea, L. corymbulosa, L. suaveolens (Sleumer 1980).

\section{Key to species of Laetia in Reserva Ducke}

1. Secondary veins $8-14$ pairs; bract subtending pedicel $0.5-1 \mathrm{~mm}$ long, broadly ovate to rectangular-ovate; sepals $2.5-3.5 \mathrm{~mm}$ long, glabrous on both sides; stamens 12-16, anthers 1.4-2 mm long. Capsule globose or trigonal-globose, width equal to or greater than length, apex rounded, mature pericarp glabrous, transversely wrinkled 1. L. procera

1. Secondary veins 4-6 pairs; bract subtending pedicel 5-7 $\mathrm{mm}$ long, basal part narrowly cylindrical, pedicel-sheathing, apical part expanded, cupular; sepals 7-8 $\mathrm{mm}$ long, densely hairy on both sides; stamens > 30, anthers 0.6-0.7 mm long. Capsule ovoid-ellipsoid, length > width, apex acute, pericarp densely hairy, not transversely wrinkled

2. L. cupulata

3.1 Laetia procera (Poepp.) Eichl. in Mart. Fl. bras. 13(1): 453. 1871.

Laetia casearioides Sagot ex Benth., J. Proc. Linn. Soc. Bot. 5 (Suppl. 2): 85. 1861. Casearia bicolor Urb., Symb. Antill. 1: 372. 1899.

Casearia belizensis Standl., Field Mus. Nat. Hit., Bot. Ser. 12: 412. 1936.

Tree, 8-30(-40) $\mathrm{m}$ tall, trunk beige to reddish- or pinkish-grey, with conspicuous lenticels. Young twigs and branchlets sulcate, very minutely puberulent (hairs just visible at $\mathrm{x}$ 10 mag.), older twigs and branchlets becoming glabrous. Lenticels on branches numerous, raised, conspicuous. Stipules $0.5-0.6 \mathrm{~mm}$ long, broadly triangular, sparsely puberulent, with ciliate and coarsely papillate margin, caducous. Petioles 6-10×1-1.5 mm, with margins more or less inrolled, very minutely puberulent (hairs visible at x 20 mag.), drying blackish. Leaves $8.5-19.5 \times 3.5-5.5 \mathrm{~cm}, 2.5-4$ times as long as broad, firm-chartaceous, long-elliptic to longoblong, pellucid-punctate/lineate, areoles finely black-dotted; base rounded to cordate, sometimes oblique; apex cuspidate-acuminate, acumen $0.4-1.2 \mathrm{~cm}$ long, extreme tip obtuse, not apiculate; lamina upper surface glabrous, slightly glossy, lower surface glabrous except for minutely puberulent midvein and secondary veins (hairs just visible at $\times 10$ mag.). Midvein raised and conspicuous below. Secondary veins $8-14$ pairs, branching from midvein at $30-60^{\circ}$. Leaf margin very shallowly serrate. Inflorescence supra-axillary, fasciculate, fewto many-flowered, adjacent fascicles sometimes coalescent. Bracts numerous, congested, forming a globose cushion at the fascicle base, each bract 0.5-1 mm long, papery, broadly ovate or rectangular-ovate, with apex apiculate or obtuse, outside sericeous, inside glabrous, margin ciliate. Pedicel in flower 5-7 mm long, extending to 10$12 \mathrm{~mm}$ after anthesis, filamentous, articulate at base, glabrous, base closely encircled by bracts. Bud globose to broadly ovoid, apex obtuse to rounded. Sepals 4-5, 2.5-3.5 mm long, connate at base, broadly ovate to suborbicular, rounded at 
apex, subpetaloid, translucent, with membranous margins, 3-veined, glabrous on both surfaces, whitish to greenish when fresh. Stamens 12-16, uniseriate; filaments 2-2.2 mm long, slender, glabrous; anthers dorsifixed towards base, 1.4$2 \mathrm{~mm}$ long, linear to narrowly triangular, glabrous, connective glabrous. Disc glabrous. Ovary ca. $1.5 \mathrm{~mm}$ long, ovoid, tapering gradually into style, glabrous. Style ca. $1.5 \mathrm{~m}$ long, stout at base, gradually tapering to flat-capitate stigma, glabrous. Pedicel in fruit 12-15 mm long. Capsule dehiscent, 10-20 mm long, trigonal-globose, in dried material sometimes almost 3-lobed by differential contraction of pericarp, rounded at base and apex, maroon when fresh, drying darkish brown to blackish, style remnant persistent at apex, perianth remnants or at least reflexed sepals persistent at base. Pericarp thin, 0.8-1 mm, laminate and resinous in cross-section, hard when dried, longitudinal median band of each carpel contracted into fine, transverse wrinkles, sometimes also with parallel, raised, thickened, horizontal bands, glabrous, with or without drops of milky-white resin; pericarp inside glabrous, glossy; valves 3 , broadly elliptic to suborbicular, with rounded apex. Seed 30-40, mostly 3-4 mm long, compressed globose-ovoid to ellipsoid, variable in shape, basal $1 / 4$ to $1 / 5$ constricted, base truncate, apex obtuse to rounded, testa markedly reticulate, minutely echinate in cross-section, covered entirely by a thin, semi-transparent membrane; aril nonfimbriate, thin, whitish or yellowish, waxy.

Habitat within Reserva Ducke: Canopy or understorey of forest, on slopes; on clay or sandy soil.

Distribution within Reserva Ducke: "floresta de platô", "floresta de vertente".

General distribution: Caribbean Is., Central America from Guatemala southwards, Amazonian South America southwards to northern Bolivia. Material from the Reserva Ducke: Assunção 234, 457; Rodrigues 6873 (not seen), 6891 (not seen), 7540 (not seen), 7584 (not seen), 7666 (not seen); Sothers 677, 706.

Additional material: BRAZIL. RORAIMA: Poste Mucajaí, Rio Mucajaí, 25.III.1971 (fr) Prance et al. 11220 (IAN INPA K). AMAPÁ: about $5 \mathrm{~km}$ southwest of mouth of Rio Ingarari, $2^{\circ} 17^{\prime} \mathrm{N}, 52^{\circ} 41^{\prime} \mathrm{W}$, 18.IX.1960 (fl) Irwin et al. 48360 (K MG NY); Rio
Araguari, Camp 13, $1^{\circ} 45^{\prime} \mathrm{N}, 25^{\circ} \mathrm{W}, 9 . X .1961$ (fl fr) Pires et al. 51608 (K MG NY). PARÁ: Santarém, km 70 da estrada do Palhão, Ramal do Caetetu, 9.IX.1969 (fl) Silva \& Souza 2526 (K MG NY).

3.2 Laetia cupulata Spruce ex Benth., J. Proc. Linn. Soc. Bot. 5 (Suppl.2): 84. 1861. Fig. 3

Tree 4-15(-20) $\mathrm{m}$ tall. Young twigs and branchlets sulcate, densely puberulent, most hairs very short, spreading, just visible at $\times 10$ mag., lenticels inconspicuous, older twigs and branchlets becoming glabrous. Stipules 3-4 mm long, narrow-triangular, adpressed-hairy, early caducous. Petioles 8-10 × 1.3-1.8 mm, grooved and narrowly winged above, very sparsely adpressed hairy, drying blackish. Leaves $11-16 \times 5.5-6.5 \mathrm{~cm}, 2-2.8$ times as long as broad, firm-chartaceous, broadly elliptic or oblong-elliptic; base broadly acute-cuneate, rounded, or truncate, sometimes very shortly attenuate at junction with petiole; apex cuspidate-acuminate, acumen $0.5-1 \mathrm{~cm}$ long, extreme tip obtuse, minutely apiculate; lamina practically glabrous above and below, except for sometimes very sparsely adpressedpubescent midvein and secondary veins, impunctate. Midvein raised and conspicuous below. Secondary veins $4-6$ pairs, at $45-60^{\circ}$. Leaf margin shallowly subcrenate to coarsely and shallowly serrate. Inflorescence axillary, fasciculate, several-flowered, in foliate or defoliate axils of young twigs. Bracts very conspicuous, 5-6 mm long, basal part (0.5-) 1-3 mm long, narrow tubular, closely sheathing pedicel base, apical part 3-4 $\mathrm{mm}$ long $\times c a$. $3 \mathrm{~mm}$ diameter, expanded and cup-shaped, both parts pubescent throughout outside, glabrous inside, persistent, drying blackish brown. Pedicel at anthesis slender, 7-10 mm long, pubescent. Sepals 5, 7-8 mm long, connate at base, broadly oblong-elliptic, rounded at apex, opaque, whitish or pinkish when fresh, inside with several close, parallel, longitudinal veins, outside densely grey-sericeous, inside greyish sericeous/puberulent mixed, especially along veins and in apical half. Stamens numerous, in 2-3 close-set rows; filaments 4-6 mm long, inequilong, slender, glabrous; anthers versatile, 
0.6-0.7 mm long, oblong-ellipsoid, glabrous, connective not swollen nor projecting, glabrous. Disc hairy. Ovary ca. $2 \mathrm{~mm}$ long, globose, densely ferruginous-sericeous, ovules 10-12 per placenta. Style 5-6 mm long, filamentous, sparsely pubescent, 3-branched at apex, each branch ca. $1 \mathrm{~mm}$ long, recurved, stigmas capitate. Pedicel in fruit 10-12 mm long, slender, cupular bract persistent. Capsule 1.3$1.5 \mathrm{~cm}$ long, dehiscent, reddish or reddish green when fresh, drying reddish-brown, ovoidellipsoid, trigonal, acute to obtuse at base, acute at apex, with style/style remnant persistent at apex, perianth remnants including reflexed sepals persistent at base; pericarp coriaceous, outside with dense indumentum of short, spreading, ferruginous hairs interspersed with longer, paler, semi-adpressed wavy hairs, pericarp inside dark yellow-brown, glossy, pilose, densely so along valve midline; valves 3 , erect, valve walls $c a$. $0.5 \mathrm{~mm}$ thick, laminate in cross-section. Seed 1 , broadly ovoid-ellipsoid, minutely and densely foveolate (not foveolate-reticulate), glabrous or with just a few scattered hairs, orange-brown; aril thin, waxy, flaky, yellowish or whitish.

Habitat within Reserva Ducke: Sub-canopy tree of lowland forest, near streams; sandy soil. Distribution within Reserva Ducke: "floresta de baixio".

General distribution: Amazonian S. America. Material from the Reserva Ducke: Souza 403 (bd), 471 (fr).

Additional material: BRAZIL. AMAZONAS: Presidente Figueredo, Estr. da UHE de Balbina, próx. ao igarapé do Barreto, $1-2^{\circ} \mathrm{S}, 59-60^{\circ} \mathrm{W}$, 31.III.1986 (fr) Ferreira et al. 7041 (INPA K MG NY); São Gabriel de Cachoeira, Rio Içana, 5.XI.1987 (bd) Rodrigues 10857 (INPA K NY).

Species recognition: Laetia cupulata can be recognised by the characteristic sheathingcupular bract subtending each pedicel. In bud the bract completely encloses the pedicel and flower and may be mistaken for a calyx, in which case the true calyx may be mistaken for a corolla. In fruit L. cupulata bears a strong, if superficial, resemblance to Casearia javitensis, the leaf shape, leaf texture, capsule shape and capsule indumentum being particularly similar. The two taxa can be distinguished by the bracts, and, if present, perianth and disc remnants, L. cupulata having cupular-sheathing bracts and many stamens with no alternating disc lobes.

\section{Xylosma \\ Xylosma G. Forst., Fl. Ins. Austr. 72. 1786.}

Shrubs or trees. Branches often armed. Stipules caducous. Leaves alternate, petiolate, penninerved, margin often toothed, teeth each with a circular apical gland on underside. Inflorescences axillary, fasciculate or shortly racemose, few-flowered. Flowers very small, unisexual or rarely bisexual. Pedicels articulate. Sepals 4-5(-7), imbricate. Petals 0. Disc extrastaminal (male flowers) or surrounding ovary (carpellate flowers), entire, undulate, or composed of several small globose to compressed-angular connate lobes. Staminate flowers: stamens few to many, filaments free, anthers minute, globose to ellipsoid, dorsifixed, rudiment of ovary 0 . Carpellate flowers: ovary 1-locular, placentas 2-3(-6), parietal, styles 2-3(-4), often very short, joined in lower part only or completely joined to form a single style column, or styles absent; stigmas semilunate to U-shaped; staminodes 0. Berry small, generally dry with thin coriaceous pericarp, seed few, aril absent.

About 95 species, ca. 50 in Central \& South America, the rest in Asia-Malesia and the Pacific. One species from the Reserva Ducke. Also from Brazilian Amazonas: $X$. benthamii, X. intermedium (Sleumer 1980).

\subsection{Xylosma tessmannii Sleumer, Notizbl. Bot. Gart. Berlin 12: 477. $1935 . \quad$ Fig. 4}

Tree to $10 \mathrm{~m}$ tall (terminal bud not seen). Trunk armed with spines to $12 \mathrm{~cm}$ long. Twig tips and young branchlets glabrous to sparsely pubescent, lenticellate, older branches glabrous, cataphylls several in transitional zone between new and old twig growth, $1.5 \mathrm{~mm}$ long, triangular to subulate, scale-like. Stipules minute, $c a .0 .2$ $\mathrm{mm}$ long, indurate, triangular. Petioles 3-7×1.2$1.5 \mathrm{~mm}$, sparsely puberulent, glabrescent. Leaves thinly chartaceous when young, becoming subcoriaceous, variable in shape and size, 9.5$15(-24) \times(3-) 4-7.5(-8.5) \mathrm{cm}, 1.7-3.5$ times 


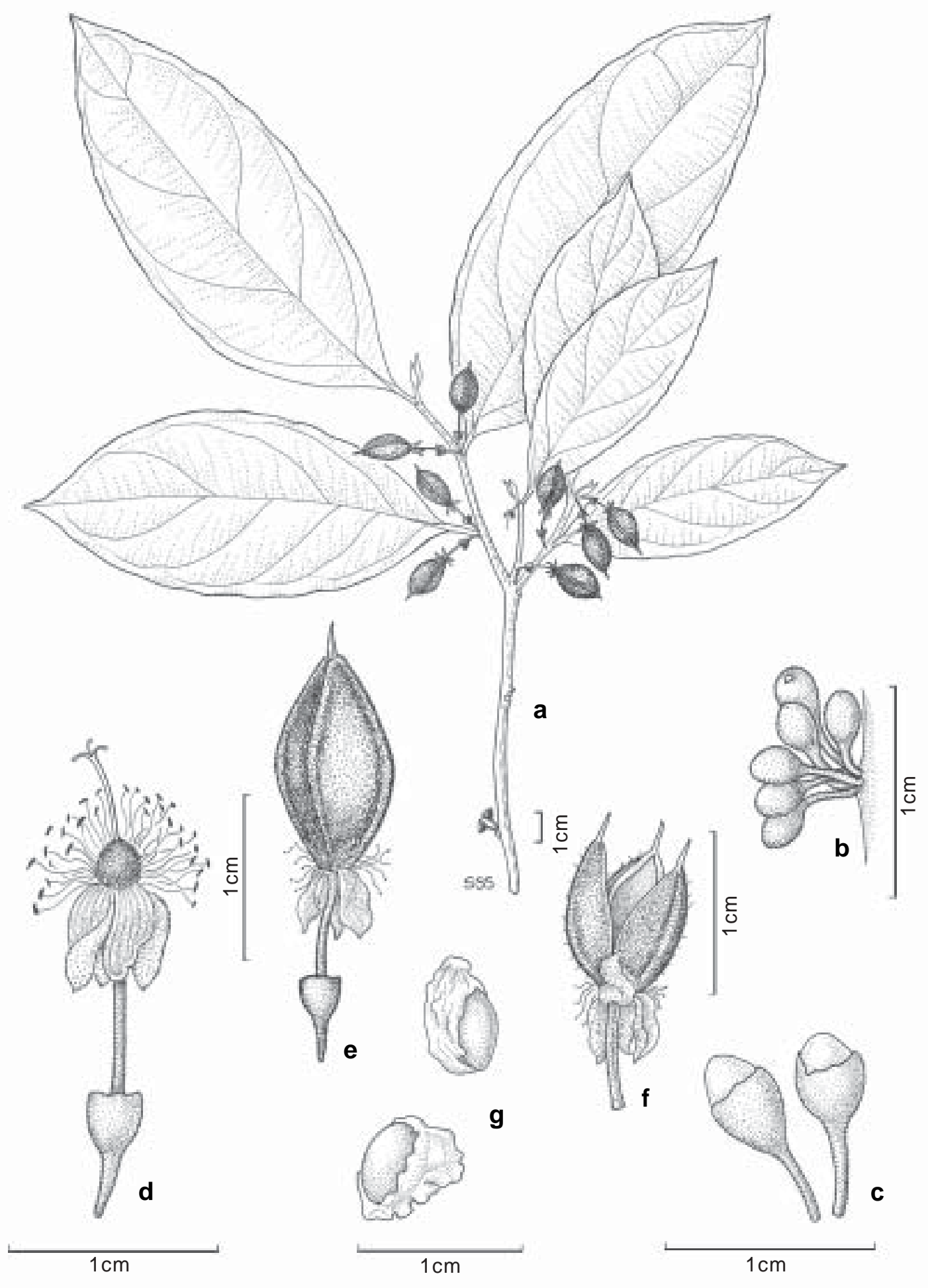

Figure 3 - Laetia cupulata - a. habit; b. young inflorescence: buds enclosed by cupular bracts; c. young inflorescence: buds with sepals emerging from cupular bracts; d. flower, with cupular bract at pedicel base; e. fruit, before dehiscence; f. fruit, after dehiscence; g. seed, with arils. (a, d-e. from de Souza 471; b. from de Souza 403; c. from Rodrigues 10857; f-g. from Ferreira 7041). Drawn by Susanna Stuart-Smith. 


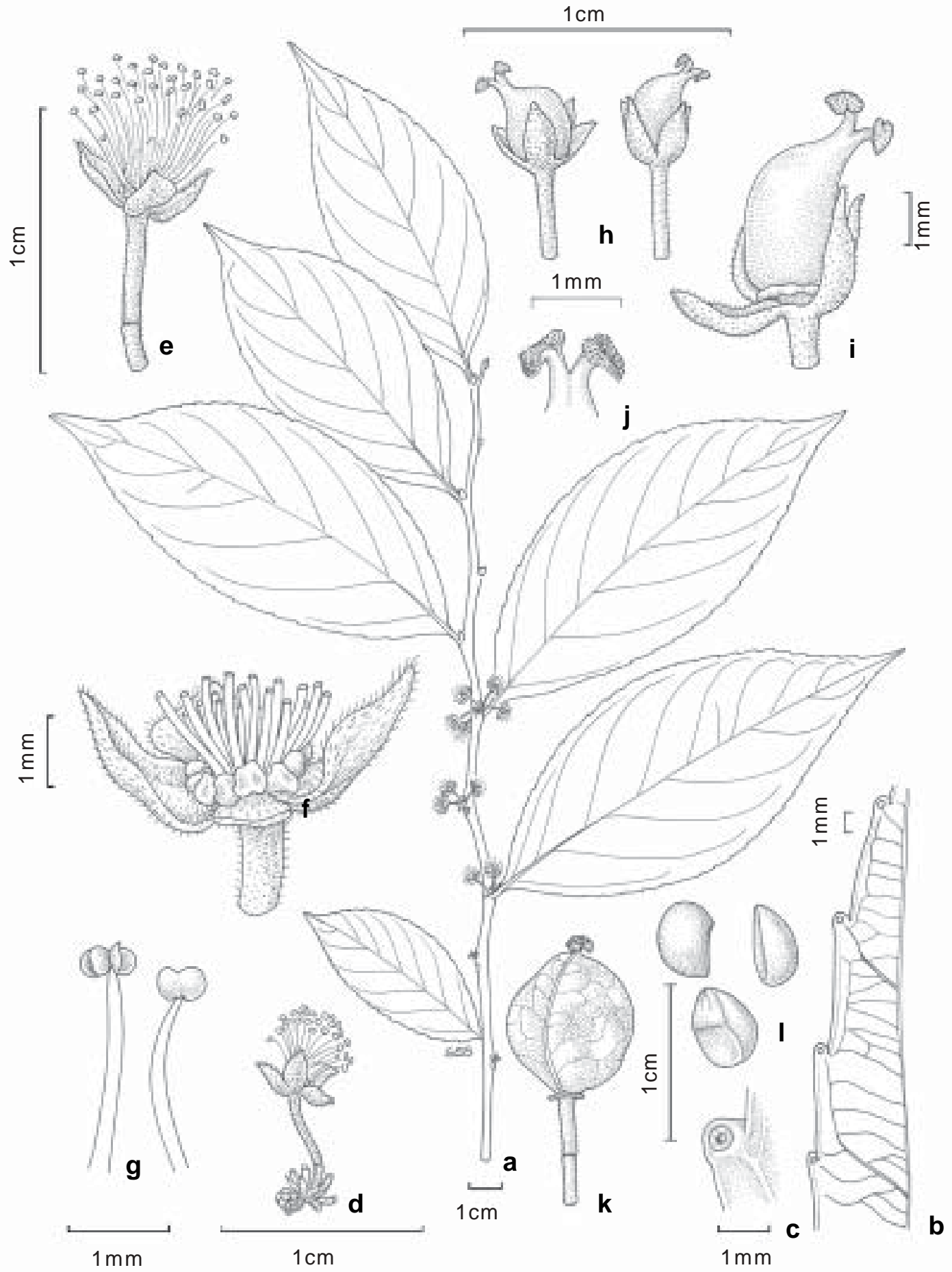

Figure 4 - Xylosma tessmannii - a. habit (male); b. leaf: underside of margin; c. gland on underside of leaf tooth; d. inflorescence (male): fascicle with one flower remaining; e. flower (male); f. flower (male): dissected, with sepal and stamens partly removed to show disc lobes; g. stamens; h. very young fruit; i. very young fruit, with sepal reflexed to show disc; j. stigmas; k. fruit; 1. seed. (a, d-g. from Klug 3026; b-c, h-j. from Krukoff 5617; k-1. from Heyde 398). Drawn by Susanna Stuart-Smith. 
as long as broad, narrowly to broadly elliptic, less often slightly obovate, base acute to obtuse with 1-2 small circular glands near junction with petiole, apex cuspidate-acuminate, acumen 1$1.5 \mathrm{~cm}$ long, extreme tip rounded to acuteapiculate, lamina minutely and densely pellucidpunctate, at least when young, glabrous on both surfaces or rarely with a few spreading hairs on venation below. Midvein raised below, often conspicuous. Secondary veins 5-8(-9) pairs, curved-ascending, branching from midvein at $35-55^{\circ}$. Leaf margin coarsely serrate-crenate, each leaf tooth with a circular, thick-bordered gland on underside. Inflorescences in foliate or defoliate axils of young twigs, short-racemose to subfasciculate, raches to $2 \mathrm{~cm}$ long, severalflowered. Cataphylls/bracts at base of inflorescence short-triangular, scale-like, $c a .1$ $\mathrm{mm}$ long, bracts towards apex of inflorescence long-triangular to subulate, $c a$. $1.5 \mathrm{~mm}$ long, all cataphylls/bracts glabrous outside, margin ciliate. Pedicels in flower 3-4 mm long in staminate flowers, slightly longer in carpellate flowers, slender, articulate within basal 1/3-1/ 2 , glabrous to sparsely puberulent. Buds globose. Sepals 4-5, 2-3 mm long, connate at base, ovate-oblong, acute to rounded at apex, apiculate or not, sometimes finely keeled, yellowish when fresh, subglabrous to puberulent on both surfaces, margin usually ciliate. Staminate flowers: disc lobes 8-10, close-set, each 0.4 $0.5 \mathrm{~mm}$ diam., polygonal, glabrous; stamens $\mathrm{ca}$. 30; filaments ca. $4 \mathrm{~mm}$ long, glabrous; anthers minute, pollen sacs subglobose, glabrous, separated quite widely by connective, the latter drying dark brown in contrast to paler pollen sacs. Carpellate flowers: disc entire or lobed, flattish or undulate; ovary narrowly ovoid, tapering gradually at apex, glabrous, with 2-3(4) placentas, styles 2-4, more or less connate, style plus stigma $0.5-0.8 \mathrm{~mm}$ long, glabrous, stigmas flattened-deltoid to semi-lunate or ushaped, erect to reflexed. Infructescences usually in defoliate axils. Pedicel in fruit 3.5-7 mm long, slender. Berry 1.5-2 cm long (Sleumer 1980), broadly ellipsoid, ovoid or globose, asymmetric at least when young, base and apex rounded, calyx, disc and style remnants persistent; pericarp thin, $0.2-0.3 \mathrm{~mm}$, coriaceous, in dried material often with 4-6 straight raised longitudinal veins/ridges and otherwise covered with raised reticulate venation, pericarp outside glabrous, yellow, red or reddish-brown when fresh, drying blackish, inside smooth, glabrous, dark brown. Seed 2-6, 4-7 mm long, ovoid, compressed, testa smooth, glabrous, yellow to red, drying pale brown to blackish.

Sleumer (1980) noted that X. tessmannii might be a luxuriant form of $X$. benthamii (Tul.) Triana \& Planchon.

Habitat within Reserva Ducke: forest on terra firma; clay soil.

Distribution within Reserva Ducke: (data not available).

General distribution: Amazonian South America.

Material from the Reserva Ducke: Prance et al. 2186 (staminate fl., abnormal?).

Additional material: BRAZIL. AMAZONAS: near mouth of Rio Embira (trib. of Rio Tarauaca), $7^{\circ} 30^{\prime} \mathrm{S}$, $70^{\circ} 15^{\prime}$ W, VI.1933 (bd) Krukoff 4781 (K NY). ACRE: near mouth of Rio Macauhan (trib. of Rio Yaco), 9²0'S, 69 W, 22.VIII.1933 (yfr) Krukoff5617 (KNY). RONDÔNIA: 0-3 km W of Rio Madeira along road Abunã to Rio Branco, 13.VII.1968 (fr) Prance et al. 5948 (INPA K NY US). GUYANA. Biara Creek, Moruka, III.1947 (fr) Fanshawe 2685, For. Dept. no. 5478(K). SURINAME. S. of Paramariba, 26.XII.1976 (fr) Heyde 398 (K U). PERU. Loreto, Balsapuerto, IV.1933 (fl) Klug 3026 (K).

Species recognition: $X$. tessmannii in fruit can be confused with Casearia, from which it can be distinguished by disc morphology and the leaf tooth foliar glands.

\section{Casearia}

Casearia Jacq., Enum. Syst. Pl. 4: 21. 1760.

Shrubs or trees. Branchlets armed or unarmed. Stipules usually early caducous. Leaves alternate, distichous, penninerved, rarely 3veined from base, often pellucid-punctate/ lineate. Inflorescences axillary or supra-axillary, usually fasciculate or glomerulate, rarely shortcymose, few- to many-flowered, in or above foliate or defoliate axils of twigs and branchlets. Bracts generally small, papery, congested around pedicel and peduncle bases, often numerous and 
then forming a small, hemi-spherical cushion, persistent at least as remnants. Pedicels articulate, often slender, basal part persistent after abscission of flower. Flowers small, $<10$ $\mathrm{mm}$ long, at least structurally bisexual, always with at least a rudimentary ovary. Calyx 4-5lobed, more or less cupular or tubular in lower part, $<10 \mathrm{~mm}$ long, imbricate, free almost to base or connate in basal $1 / 4$ to $2 / 3$ to form a calyx tube, lobes erect or reflexed at anthesis. Petals 0. Disc more or less cup-shaped, adnate to inside of calyx tube, lobed, rim and lobes free; disc lobes equal in number to stamens and alternating with them or seemingly in a separate row inside the staminal row, linear to narrowly oblong or narrowly clavate, 1/4-3/4 × length of stamen filaments. Stamens $c a .5-20$, perigynous, uniseriate, free, attached to rim of disc either in the sinuses between the disc lobes or seemingly in a separate, single row outside of the disclobe row; filaments generally slender, often alternately inequilong; anthers usually small, subglobular to ellipsoid, versatile, dehiscent by longitudinal slits, connective often swollen or with a hairy or glabrous apical gland. Ovary 1locular; placentas 3, parietal, non-intrusive, multiovulate. Style simple or 3-branched at apex, stigma(s) capitate. Fruit usually a capsule, less often baccate, dry or fleshy, indehiscent or dehiscent by (2-)3(-4) valves, often 3-angled, 0.5-7 cm long; seed 1-ca. 20, sarcotesta and aril present, the latter usually distally fimbriate.

About 180 species, pantropical. Eleven species from the Reserva Ducke. Also from Brazilian Amazonas: $C$. aculeata, $C$. commersoniana, C. decandra, C. fasciculata, C. mariquitensis, C. neblinae, C. obovalis, C. spruceana, C. tenuipilosa, C. uleana.

\section{Key to species of Casearia in flower in Reserva Ducke}

1. Terminal bud and inflorescence-bearing axils resin-coated or if not then leaves $17-28 \mathrm{~cm}$ long, glabrous below, and pedicels $<1 \mathrm{~mm}$ long

1. C. resinifera

1. Terminal bud and inflorescence-bearing axils not resin-coated, character combination not as above. 2. Style 3-branched.

3. Leaves 7-10 cm long, calyx $1.5-2.2 \mathrm{~mm}$ long, disc lobes in same row as stamens, ovary glabrous ................................................ 1. C. sylvestris var. sylvestris

3. Leaves (7.5-)12.5-22 cm long, calyx 4-5 mm long, disc lobes intrastaminal, ovary densely sericeous

2. C. javitensis

2. Style entire.

4. Underside of mature leaf densely tomentose.

5. Leaves $6-9.5 \times 1.8-2.7 \mathrm{~cm}$, pedicels $<0.5 \mathrm{~mm}$ long, sepals densely sericeous outside 6. C. grandiflora

5. Leaves $12-18 \times 4.5-6 \mathrm{~cm}$, pedicels $8-10 \mathrm{~mm}$ long, sepals densely and minutely tomentose outside 7. C. arborea forma

4. Underside of mature leaf glabrous to variously hairy but never tomentose.

6. Sepals strongly reflexed at anthesis 8. C. duckeana

6. Sepals erect to spreading at anthesis, not reflexed.

7. Stamen filaments densely long-villous or long-hispid (stamen filaments not to be confused with alternating disc lobes).

8. Anther connective not swollen nor projecting, more or less glabrous, at least without long wiry hairs; leaves often drying greyish-green

3. C. combaymensis

8. Anther connective apiculate, very long-hispid, the hairs erect, wiry, about as long as the anther itself and projecting conspicuously beyond the anther; leaves often drying darkish brown....

9. C. pitumba

7. Stamens filaments glabrous or with few hairs towards base. 
9. Tertiary veins more or less reticulate (see leaf underside $\times 10$ mag.), 1-4 mm apart, not especially close set, angled and branched (i.e. not straight), not especially parallel to one another, running at various angles to midvein, not percurrent; ovary sparsely hairy to glabrous; calyx 2$3.5 \mathrm{~mm}$ long

5. C. ulmifolia

9. Tertiary veins strongly percurrent (see leaf underside), i.e. ladder-like, close set (1-2 mm apart), more or less straight, parallel to one another, all running at ca $90^{\circ}$ to midvein; calyx 5-7.5 mm long. 10. Underside of mid- and secondary veins of mature leaves spreading-pilose; anther 0.5$0.6 \mathrm{~mm}$ long, broadly ellipsoid, connective globose, swollen, sparsely bearded or glabrous ....

4. C. manausensis

10. Underside of mid- and secondary veins of mature leaves glabrous; anther $0.8-1 \mathrm{~mm}$ long, ovoid-triangular, connective narrowly lanceolate, not swollen, glabrous

10. C. negrensis

\section{Key to species of Casearia with mature fruit in Resera Ducke}

1. Terminal bud and axillary bract cushions at bases of infructescence resin-coated, pericarp outer surface with or without resin drops

1. C. resinifera

1. Terminal bud and axillary bract cushions (i.e. bases of infructescence) not resin-coated, pericarp outer surface without resin drops.

2. Outer surface of capsule densely velutinous or tomentose throughout.

3. Mature capsule $1.5-2 \mathrm{~cm}$ long (excluding style remnant), densely greyish- or brownishvelutinous, pedicel $7-10 \mathrm{~mm}$ long; leaves mostly $7-11 \mathrm{~cm}$ long, secondary veins $3-5$ pairs

8. C. duckeana

3. Mature capsule $0.8-1.2 \mathrm{~cm}$ long (excluding style remnant), finely and densely reddishbrown tomentose with interspersed, longer, straight, white hairs; pedicel $3-5 \mathrm{~mm}$ long; leaves (7.5-)12.5-22 cm long, secondary veins (5-)6-9 pairs 2. C. javitensis

2. Outer surface of capsule sparsely hairy or glabrous (sometimes densely hairy at apex), not velutinous, not tomentose.

4. Underside of mature leaf densely tomentose.

5. Leaves $6-9.5 \times 1.8-2.7 \mathrm{~cm}$, pedicels $<0.5 \mathrm{~mm}$ long, sepals densely sericeous outside

6. C. grandiflora

5. Leaves $12-18 \times 4.5-6 \mathrm{~cm}$, pedicels $8-10 \mathrm{~mm}$ long, sepals densely and minutely tomentose outside

7. C. arborea forma

4. Underside of mature leaf glabrous or variously hairy, not tomentose.

6. Mature capsule enclosed to $c a$. half-way or more by persistent erect sepals, small, $<1 \mathrm{~cm}$ long, dry.

7. Tertiary veins (see leaf underside $\times 10$ mag.) percurrent, i.e. ladder-like, close-set (1-2 mm apart), more or less straight, parallel to one another, all running at $c a .90^{\circ}$ to midvein, capsule $8-9 \mathrm{~mm}$ long, seed $4-10(-13)$.......

4. C. manausensis

7. Tertiary veins (see leaf underside $\times 10$ mag.) more or less reticulate, i.e. $1-$ $4 \mathrm{~mm}$ apart, not especially close set, angled and branched (i.e. not straight), not especially parallel to one another, running at various angles to midvein, not percurrent; capsule 3-5.5 mm, seed 1-4 5. C. ulmifolia

6. Mature capsule not enclosed by sepals, dry or fleshy, up to $6 \mathrm{~cm}$ long.

8. Mature capsule $<1.2 \mathrm{~cm}$ long, pericarp veined, verrucose, transversely wrinkled, dehisced valves widely-spreading to reflexed, bi-colouration of inner surface forming Y-shape .................. 1. C. sylvestris var. sylvestris

8. Mature capsule $2-6 \mathrm{~cm}$ long, valves not as above. 
9. Mature capsule ellipsoid, 3-6-angled by strongly-raised longitudinal ridges marking the valve keels and sutures, outer surface of pericarp often irregularly veined 10. C. negrensis

9. Mature capsule globose, outer surface of pericarp smooth, minutely and shallowly verrucose, not veined.

10. Leaves 14-28 $\times 4.5-9 \mathrm{~cm}, 3-3.7$ times as long as broad, chartaceous, oblong to longoblong, less often long-elliptic, usually drying darkish brown; secondary veins 10-14(-17) pairs, branching from midvein at broad angle $\left(60-80^{\circ}\right)$...................... 11. C. resinifera

10. Leaves $7-13.5 \times 3.5-9 \mathrm{~cm}, 1.5-3$ times as long as broad, chartaceous to subcoriaceous, dried colour various; secondary veins 4-8(-10) pairs, branching from midvein at narrower angle $\left(30-60^{\circ}\right)$.

11. Mature capsule $2.5-3 \mathrm{~cm}$ diameter, drying dark brown, valve sutures marked by three fine impressed lines; mature leaves usually chartaceous, usually drying dark brown 9. C. pitumba

11. Mature capsule (3-)5-7 cm diameter, drying pale brown, greyish-brown or orangebrown, longitudinal impressed lines absent; mature leaves subcoriaceous, drying pale-, greenish- or greyish-brown 3. C. combaymensis

Casearia Sect. Crateria Benth. in Hooker, Journ. Bot. 4: 110. 1841; Sleumer, Fl. Neotropica 22 : Flacourtiaceae, 387. 1980.

Style 3-branched at apex; stamens 10; disc lobes in same row as and alternating with stamens; capsule dry, small, 1-few seeded.

5.1 Casearia sylvestris Sw. var. sylvestris, Fl. Ind. Occid. 2: 752.1798.

Shrub or tree to $20 \mathrm{~m}$ tall, trunk reddishbrown to dark grey. Twig tips and youngest branchlets slightly sulcate, puberulent to glabrous Lenticels sparse to absent on younger branchlets, sparse to dense on older branchlets. Stipules $c a .0 .5 \mathrm{~mm}$ long, broadly ovate or triangular, acute at apex, hairy, more or less persistent. Petioles 3-5 $51-1.2 \mathrm{~mm}$, grooved above, becoming glabrous. Leaves $7-10 \times 2.5-$ $4.5 \mathrm{~cm}, 2-3$ times as long as broad, chartaceous to subcoriaceous, elliptic to oblong-elliptic, base acute to rounded, asymmetric, apex cuspidateacuminate, acumen $1-1.5 \mathrm{~cm}$ long, extreme tip obtuse, minutely apiculate; lamina densely to obscurely pellucid-punctate/lineate, glabrous above, practically glabrous below. Midvein raised below, conspicuous or not. Secondary veins (6-)7-9(-10) pairs, weakly curvedascending for most of their length, branching from midvein at $60-70^{\circ}$, slightly raised above and below, not very conspicuous on either surface leaf apex. Leaf margin minutely and distantly serrate throughout. Inflorescences sessile, fasciculate, few- to many-flowered, in foliate or defoliate axils of young twigs. Bracts $0.5-1 \mathrm{~mm}$ long, triangular-ovate, papery, outside densely puberulent. Pedicel in flower $2-3.5 \mathrm{~mm}$ long, slender, puberulent, articulate in basal 1/3. Bud obovoid, apex rounded. Flower very small, strongly and sweetly aromatic. Calyx 1.5-2.2 $\mathrm{mm}$ long, fused in basal 1/3-1/2 to form a short tube, calyx lobes ('sepals') 5 , erect at anthesis, oblong to oblong-obovate with rounded apex, outside puberulent, pale yellow to whitish or greenish when fresh, becoming purplish in fruit, inside minutely pilose, margin ciliate. Stamen filaments $1-1.5 \mathrm{~mm}$ long, sparsely puberulent; antherca. $0.2 \mathrm{~mm}$ long, broadly globose-oblong, practically glabrous, connective with small, swollen, glabrous apical gland. Disc cup sparsely pilose inside; disc lobes $0.5 \mathrm{~mm}$ long, narrowly oblong, densely white-villous at apex. Ovary ovoid to broadly ellipsoid, slightly ridged longitudinally, glabrous, placentas each with 3-6 ovules. Style $0.8-1 \mathrm{~mm}$ long, sparsely pilose, apical branches ca. $0.2 \mathrm{~mm}$ long, recurved, pilose. Pedicel in fruit 3-4 mm long, slender. Capsule $0.5-0.7 \mathrm{~cm}$ long, subglobose to 3-angled, not enclosed by calyx; pericarp fleshy, outside glabrous, veined, verrucose, transversely wrinkled, yellowish to reddish-purple or brownish when fresh, drying brown, valves at dehiscence widely spreading to strongly reflexed, the bi-colouration of the 
inner surface forming a distinctive Y-pattern; perianth remnants persistent at base of capsule. Seed 6-10, flattened-ellipsoid/ovoid or polygonal, 2-2.3 × ca. $1.5 \mathrm{~mm}$, testa foveolate-reticulate, drying brown; aril substantial, irregularly folded and lobed, waxy, reddish when fresh.

According to Sleumer (1980) the flowers have a disagreeable smell.

Habitat within Reserva Ducke: understorey tree of slopes and plateau; on clay.

Distribution within Reserva Ducke: "floresta de platô", "floresta de vertente".

General distribution: Central and South America from Mexico to Argentina.

Material from the Reserva Ducke: Assunção 224; Costa 313; Hopkins 1569; Rodrigues 5340 (not seen), 5418 (not seen); Sothers 507, 508; Vicentini 1003.

Species recognition: In flower: combination of very small flower size $(<2.5 \mathrm{~mm}$ long), clearly visible pedicels $2-3.5 \mathrm{~mm}$ long, 3branched style and leaf size, shape and indumentum. In fruit: small ( $<8 \mathrm{~mm}$ long) glabrous capsules not enclosed by sepals.

Casearia Sect. Piparea (Aublet) Benth. in Hooker, Journ. Bot. 4: 110. 1841; Sleumer, Fl. Neotrop. 22 : Flacourtiaceae, 402. 1980.

Style 3-branched at apex; stamens 10-25; disc lobes in a row within the row of stamens; capsule dry; seed generally few, hairy.

5.2 Casearia javitensis Kunth in H. B. K., Nov. Gen. Sp. Pl. 5: 366, t. 479. 1823.

Shrub or small tree to $10(-15) \mathrm{m}$ tall, trunk light-brown to chestnut-brown. Youngest twigs sulcate, densely puberulent, older twigs and branchlets becoming glabrous. Lenticels inconspicuous. Stipules 2-3 mm long, narrowly triangular, acute at apex, adpressed-hairy, caducous or shortly persistent. Petioles 4-12 $\times 1.5-2.5 \mathrm{~mm}$, grooved and narrowly winged in apical half above, rounded towards base, densely puberulent (sometimes minutely), often drying blackish. Leaves variable in size and shape, (7.5-)12.5-22 × (4-)4.5-8.5 cm, (1.5-) 2.5-3 times as long as broad, thinly coriaceous, less often chartaceous, mostly elliptic to oblongelliptic, symmetric, base broadly acute to rounded, rarely truncate, sometimes shortly attenuate, asymmetric or not, apex cuspidateacuminate, acumen (0.5-)1-1.8 cm long, extreme tip acute to obtuse, apiculate; lamina practically impunctate, glabrous on both surfaces except for sometimes puberulent midand secondary veins. Midvein raised below, conspicuous. Secondary veins (5-)6-9 pairs, ascending, fairly straight in basal $2 / 3$, more strongly curved in apical $1 / 3$, branching from midvein at $40-60^{\circ}$, slightly impressed above, raised and conspicuous below, sparsely puberulent to glabrous on both surfaces. Leaf margin coarsely serrate to subentire. Inflorescences axillary or supra-axillary, sessile, fasciculate, few- to many-flowered, in or just above foliate or defoliate axils of young branchlets. Bracts $0.5-1.5 \mathrm{~mm}$ long, ovate, papery, apiculate, sericeous outside, glabrous inside. Pedicel in flower 4-6 mm long, slender, densely puberulent, articulate in basal 1/2. Bud globose-ovate, apex acute to obtuse. Calyx 4-5 $\mathrm{mm}$ long, fused in basal 1/5-1/4 to form a short tube, whitish to greenish when fresh, calyx lobes ('sepals') 5, strongly reflexed at anthesis, ovateoblong, apex obtuse to rounded, both surfaces with dense indumentum of short, crimped hairs mixed with coarser, longer, straight adpressed hairs. Stamens 15-16; filaments 4-5 mm long, glabrous or with a few spreading hairs; anther ca. $0.5 \mathrm{~mm}$ long, broadly globose-oblong, glabrous, connective not swollen nor apiculate, glabrous. Disc cup inner surface very sparsely pilose to glabrous; disc lobes 1.7-2 mm long, narrow oblong, densely white- to yellow- or ferruginous-lanuginose on both faces. Ovary ovoid-globose, densely sericeous, ovules several per placenta. Style 5.5-6 mm long, glabrous except adpressed hairy towards base, apical branches ca. $0.5 \mathrm{~mm}$ long, spreading, not recurved, stigmas capitate, glabrous. Pedicel in fruit 3-5 mm long, slender, densely pubescent. Capsule 0.8-1.2 cm long (excluding style remnant), globose to ellipsoid, sometimes 3-angled or 3-ribbed, reddish- to purplish-brown when fresh and when dried; pericarp thincoriaceous, $c a$. $0.5 \mathrm{~mm}$ thick including outer indumentum, outside finely and densely reddish 
to reddish-brown tomentose with interspersed, longer, straight, white hairs, these denser at fruit apex; pericarp inside straw-yellow to orangebrown, glossy, appearing almost woody, more or less obscured by dense yellow to ferruginous lanuginose indumentum; valves after dehiscence erect to slightly spreading, each conduplicate, sometimes appearing keeled, extreme apex acuminate by persistent style-remnant; perianth remnants persistent at capsule base, not enclosing capsule. Seed 1-2(-3), relatively large, 6-7 mm diameter, compressed-globose, testa reddish brown, minutely foveolate $(\times 10$ mag.), white-pilose; aril substantial, irregularly folded and lobed, waxy, white when fresh, drying pale yellowish-brown.

Habitat within Reserva Ducke: understorey tree; mostly on clay.

Distribution within Reserva Ducke: "floresta de baixio", "floresta de vertente".

General distribution: South America, from Venezuela southwards to Bolivia.

Material from the Reserva Ducke: Assunção 442, 482; Costa 717; Sothers 954.

Assunção 442 has some adjacent inflorescences coalescent, amongst these coalescent inflorescences are flowers that seem to be functionally staminate, the ovary and style being present but rudimentary. Other flowers with developing fruit have seemingly normal stamens. Species recognition: In flower: combination of relatively large, thinly coriaceous leaves, strongly reflexed sepals, 3-branched style, and disc lobes that form a dense villous mass within the ring of stamens. C. duckeana also has strongly reflexed sepals but has smaller, chartaceous leaves (7-11 cm long), entire style and more separate disc lobes. According to Sleumer (1980) C. javitensis in flower is indistinguishable from $C$. commersoniana Cambess., a species commonly occurring in the same areas as $C$. javitensis but not yet known from the Reserva Ducke with any certainty. C. javitensis in fruit: shortly pedicellate, smallish, hairy capsules, indumentum reddish-brown, tomentose, with longer, straighter, paler hairs interspersed. $C$. duckeana also has densely hairy capsules but these are long-pedicellate $(7-10 \mathrm{~mm})$, greyvelutinous, and 1.5-2 cm long. C. javitensis in fruit bears a remarkable resemblance to Laetia cupulata (see that species).

Casearia Sect. Casearia Jacq., Enum. Pl. Car. 4, 21. 1760. Sleumer, Fl. Neotropica 22: Flacourtiaceae, 289. 1980.

Style undivided; disc lobes in the same row as and alternating with stamens.

Group Singulares sensu Sleumer in Fl. Neotropica 22: Flacourtiaceae, 289. 1980.

5.3 Casearia combaymensis Tul., Ann. Sci. Nat., Bot. Ser 3, 7: 362. 1847.

Casearia subopaca Triana \& Planchon, Ann. Sci. Nat., Bot. Ser. 4, 17: 110. 1862.

Casearia singularis Eichl. in Mart. Fl. bras. 13(1): 473, t.95. 1871.

Shrub or tree to $12 \mathrm{~m}$ tall. Twigs and branchlets sulcate/ridged, twig tips with a few adpressed hairs, older twigs and branchlets glabrous. Lenticels few to quite numerous on younger twigs and branchlets. Stipules 5-6 mm long, linear-subulate, acute at apex, sparsely adpressed-hairy, early caducous. Petioles 3-6 $\mathrm{x} c a .1 \mathrm{~mm}$, shallowly grooved above, glabrous, margins inrolled, narrowly winged. Leaves (7-) 8-13 × (3.5-)4.5-5.5 cm, 1.5-3 times as long as broad, chartaceous to subcoriaceous, narrowly to broadly elliptic, oblong or slightly obovate, symmetric, base usually narrowly to broadly acute, less often rounded, rarely truncate, apex obtuse to cuspidate-acuminate, acumen when present up to $1 \mathrm{~cm}$ long, extreme tip acute to rounded; lamina pellucid-punctate/ striate, often drying pale brown, greyish-brown or greyish-green, glabrous above, glabrous to very sparsely adpressed-pilose below, the hairs mostly confined to mid- and secondary veins. Midvein raised below, conspicuous to very much so. Secondary veins 4-6 pairs, curved-ascending, branching from midvein at $40-60^{\circ}$, finely raised on both surfaces, apical pairs more obscure. Leaf margin shallowly serrate throughout except almost entire towards base. Inflorescences sessile, fasciculate, several- to many-flowered, in foliate or defoliate axils of older branchlets. 
Bracts firm to papery, to $2 \mathrm{~mm}$ long, ovate, cuspidate at apex, puberulous outside. Pedicel in flower (3-)5-7 mm long, slender, densely pubescent, articulate within basal 1/2-1/3. Bud ovoid, acute at apex. Calyx 4.5-7 mm long, fused in basal $1 / 5-1 / 3$ to form a narrowly cylindrical tube, whitish, pale yellow or greenish when fresh, calyx lobes ('sepals') 5 , erect to spreading at anthesis, broadly ovate to narrowly oblong or narrowly triangular, with apex obtuse to rounded, hooded, outside densely short-adpressed hairy, inside densely puberulent. Stamens 10, filaments markedly inequilong, white-villous, longer filaments slender, $3 \mathrm{~mm}$ long, shorter filaments ca. $0.5 \mathrm{~mm}$ long; anthers subglobose, $0.4-0.6 \mathrm{~mm}$ long, glabrous, connective not swollen nor projecting, glabrous. Disc cup glabrous inside; disc lobes $0.8-1 \mathrm{~mm}$ long, linear to narrowly oblong, glabrous to sparsely villous. Ovary globose, glabrous or with a few hairs at apex, ovules several per placenta. Style ca. $0.5 \mathrm{~mm}$ long, glabrous, stigma oblong-capitate, papillose at apex ( $\times 20 \mathrm{mag}$.). Pedicel/peduncle in fruit stout, woody. Berry (3-)5-6(-7) cm diameter, globose, pericarp $0.2-0.7 \mathrm{~mm}$ thick, firm, outside minutely and shallowly verruculose, glabrous, greenish to orange-yellow when fresh, drying pale brown, greyish-brown or orange-brown, inside glabrous; perianth remnants caducous. Seed several, in gelatinous pulp (Sleumer 1980), relatively large, ca. $2 \times 1.5 \mathrm{~cm}$, compressed ovoid to ovoid-trigonal, base truncate, apex obtuse, testa smooth, not foveolate-reticulate; aril membranous.

Habitat within Reserva Ducke: Forest on terra firme; on clay or sandy soil.

Distribution within Reserva Ducke: (no data). General distribution: South America from Venezuela southwards to Bolivia.

Material from the Reserva Ducke: Rodrigues 4982, 6765, 6871.

Additional material: BRAZIL. AMAZONAS: Mun. Humayta, near Tres Casas, 14.IX-11.X.1934 (bd plus yfl) Krukoff 6290 (K NY); Rio Acre, VIII.1911 (fl) Ule 9726 (K). GUYANA. Labbakabra Ck., Tiger Ck., Essequibo R., 26.VIII.1937 (fl) Sandwith 1215 (K). Species recognition: In flower: often with combination of subcoriaceous leaves $8-13 \mathrm{~cm}$ long, pedicellate flowers, relatively narrow, erect sepals and glabrous anthers. C. combaymensis is variable in leaf size, shape and texture and can resemble $C$. pitumba, the only other Reserva Ducke Casearia with superficially similar flowers, but $C$. pitumba has long-villous anthers. In dried material, the leaves of $C$. combaymensis are often (but not always) a dull greyish- or greenish-brown whereas those of C. pitumba often dry a slightly glossy dark brown. In fruit: combination of large $(5-6 \mathrm{~cm}$ diam.), globose, glabrous fruit and by leaf size and texture. C. pitumba also has relatively large (2.5-3 cm diam.), globose, glabrous capsules and forms of $C$. combaymensis with smallish, immature fruit and less coriaceous leaves can be difficult to differentiate from $C$. pitumba. The fruit of both species is yellowish-green to orange at maturity (Sleumer 1980), although that of $C$. pitumba usually dries dark brown and often has the valves marked by 3 fine longitudinal ridges, whereas C. combaymensis fruit dries pale brown, greyish-brown or orange-brown and has no longitudinal ridges. The two species can sometimes be differentiated by the form of the persistent style remnant, the style base of C. pitumba often forming a small but fairly conspicuous densely hairy apicula at the apex of the fruit; in $C$. combaymensis the style base is glabrous to sparsely hairy and does not constitute a conspicuous remnant in fruit.

Group Arboreae sensu Sleumer in Fl. Neotropica 22: Flacourtiaceae, 289. 1980.

Calyx lobes or sepals overlapping considerably in flower, remaining erect after anthesis, enclosing the small capsule half-way or more.

5.4 Casearia manausensis Sleumer, Fl. Neotrop. 22: Flacourtiaceae, 321 (1980).

Fig. 5

Shrub or tree to $7 \mathrm{~m}$ tall, trunk light-brown, beige-chestnut or beige-grey. Twig tips slightly ridged/sulcate, densely spreading-pilose with pale yellow-brown hairs, becoming glabrous. Older branchlets greyish. Lenticels numerous, densely set, conspicuous or not. Stipules 5.5- 
$6.5 \mathrm{~mm}$ long, long-ovate, acute at apex, pilose, early caducous; stipule scar narrow-elliptic, $c a$. $1 \times 0.3 \mathrm{~mm}$ long, ascending-slanted, at younger nodes with a dense line of hairs along upper scar-margin. Petioles $2-3 \times 0.5-0.8 \mathrm{~mm}$, shallowly rounded above, not winged, pilose. Leaves $6.5-9.5 \times 2.2-3.5 \mathrm{~cm}, 2.5-3.5$ times as long as broad, chartaceous, oblong, oblongovate or oblong-elliptic, more or less symmetric; base broadly acute to rounded, shortly attenuate or not; apex cuspidate-acuminate, acumen $0.5-1 \mathrm{~cm}$ long, extreme tip obtuse, minutely apiculate; lamina densely pellucid-punctate/ lineate, upper surface glabrous except for pilose midvein, lower surface sparsely spreading pilose throughout. Midvein impressed above, below raised, more or less conspicuous. Secondary veins 5-8 pairs, curved-ascending, branching from midvein at $40-60^{\circ}$, impressed above, raised and well-defined below, tertiary veins strongly percurrent, i.e. ladder-like, close set (up to 1-2 mm apart), more or less straight, parallel to one another, all running at ca $90^{\circ}$ to midvein. Leaf margin finely serrate throughout, teeth sometimes elongate. Inflorescences very shortly pedunculate, glomerulate, few- to many-flowered, in foliate or less often defoliate axils of young twigs, peduncle $0.5-1.5 \mathrm{~mm}$ long, pilose. Bracts numerous, ca. $0.5 \mathrm{~mm}$ long, broadly triangular, papery, pilose outside, ciliate. Pedicel short, 1-1.5 mm long, articulate near apex, pubescent. Bud rhomboid to ellipsoid, broadly ellipsoid just before opening, apex obtuse to rounded. Calyx 6-7.5 mm long, whitish to pale yellow when fresh at first, becoming reddish in fruit, fused in basal 1/3 to form a short tube, calyx lobes ('sepals') 5, oblong-ovate, with apex rounded, hooded, adpressed-puberulent, inside minutely puberulent (view at $\times 25$ mag.). Stamens 10 ; filaments alternately inequilong, 2-2.5 mm long, straight, slender, glabrous or with a few scattered hairs; anther 0.5-0.6 mm long, broadly ellipsoid; connective globose, swollen, sparsely bearded or glabrous. Disc cup inner surface with a few long, scattered hairs or glabrous except for villous free rim; disc lobes
1.5-2 mm long, narrowly oblong, outer face adpressed-pubescent, inner face very densely long-villous throughout. Ovary ca. $2 \mathrm{~mm}$ long, narrowly ovate, tapering gradually into base of style, densely sericeous, ovules ca. 10 per placenta. Style 4-4.5 mm long, tapering gradually to apex, densely sericeous in basal half, glabrous towards apex; apex capitate, densely and shortly bearded. Pedicel in fruit ca. $3 \mathrm{~mm}$ long, slender, articulate near middle. Capsule 8-9 mm long, enclosed more than half way by persistent calyx, broad ovoidellipsoid before dehiscence, 3-angled, base obtuse to rounded, apex acute; pericarp outside sparsely adpressed-pubescent throughout, sericeous at apex towards persistent styleremnant, reddish-purple when fresh; inside glabrous; valves at dehiscence semi-spreading and recurved at apex, becoming conduplicate, valve wall $c a .1 \mathrm{~mm}$ thick in apical half, irregularly reticulate in cross section, resinous. Seed ca. 20, $34 \mathrm{~mm}$ long, irregularly ellipsoid to compressed-polygonal, basal 1/5-1/4 often constricted, base truncate, apex obtuse to rounded, testa foveolate-reticulate, appearing echinate in cross-section, covered entirely by a thin, semi-transparent membrane, orangebrown; aril substantial, irregularly folded, with long, linear extensions, waxy, white when fresh, orange-brown when dried.

Habitat within Reserva Ducke: low forest on slopes or near streams; sandy or sandy-clay soil. Distribution within Reserva Ducke: "floresta de baixio", "floresta de vertente". General distribution: South America: Brazil, around Manaus.

Material from the Reserva Ducke: Monteiro 47 (not seen); Ramos 1875; Rodrigues 7886 (not seen); Santos 885; Sothers 296, 816, 876; Souza 146 (not seen).

Species recognition: In flower: smallish oblong-elliptic leaves, secondary veins impressed above, tertiary veins percurrent, leaf underside spreading-pilose throughout, small flowers, ovary densely sericeous, style unbranched. In fruit: leaf characters as above, small fruit more than half-way enclosed by sepals. 


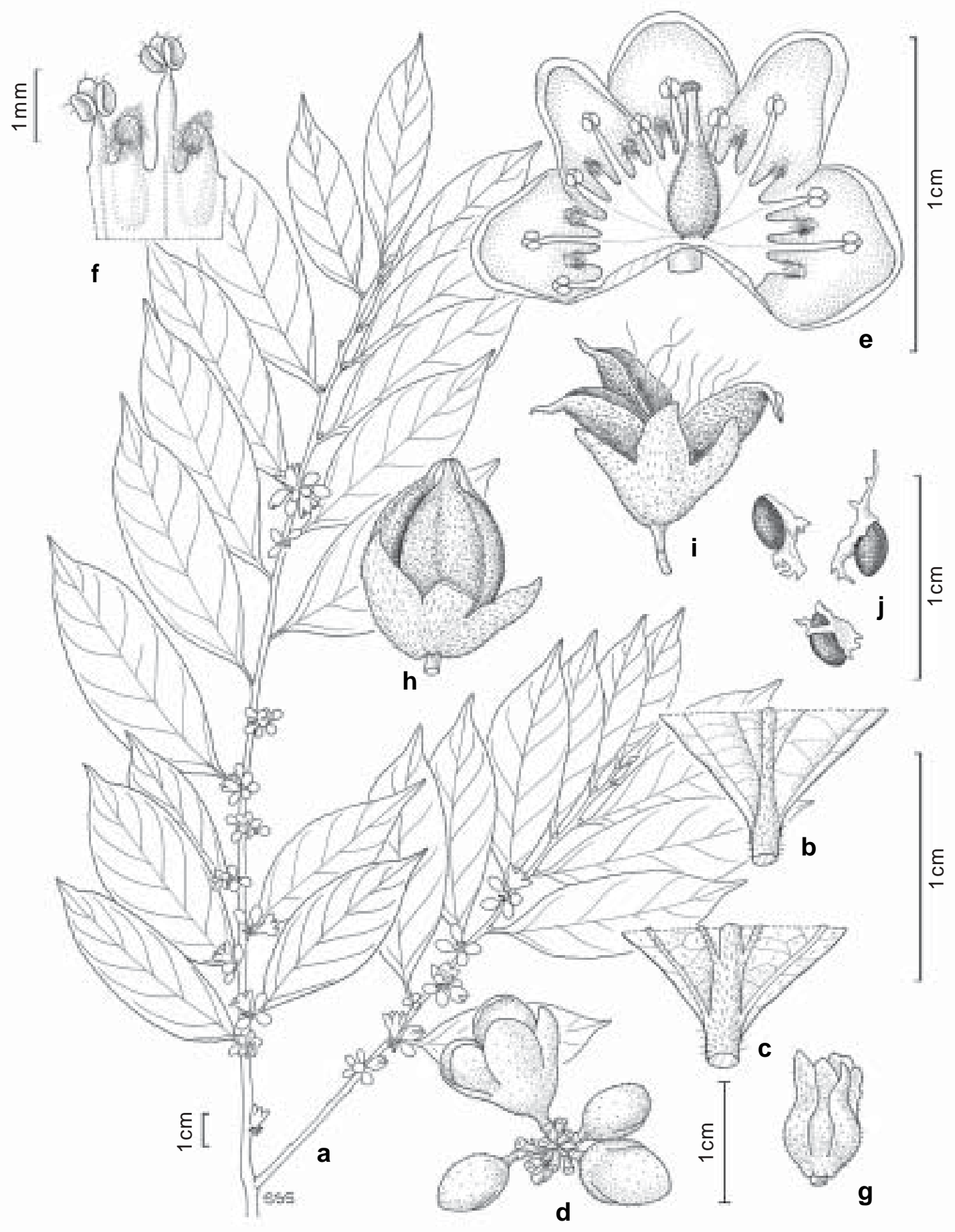

Figure 5 - Casearia manausensis - a. habit; b. leaf base, upper surface; c. leaf base, lower surface; d. inflorescence; e. dissected flower; f. part of disc, showing disc lobes and stamens; g. fertilised flower; h. fruit, before dehiscence; i. fruit, after dehiscence, with linear aril-extensions exserted; j. seed, with arils. (a-f. from Assunção 355; g. from Sothers 296; hj. from Sothers 816). Drawn by Susanna Stuart-Smith. 
5.5 Casearia ulmifolia Vahl ex Vent., Choix Pl. 46, in obs. 1808.

Casearia petraea Benth. in Hooker, J. Bot. (Hooker) 4: 111. 1841; Eichl., Mart. Fl. bras. 13(1): 477. 1871.

Casearia blanchetiana Miq., Linnaea 22: 801. 1849; Eichl., Mart. Fl. bras. 13(1): 475. 1871.

Small tree to $15 \mathrm{~m}$ tall, trunk beige (terminal bud not seen). Twig tips sulcate, twig tips and youngest branchlets sparsely to densely pilose or crisped-pilose, becoming glabrous. Lenticels conspicuous, densely set throughout. Stipules 3-5 mm long, linear, narrowly acute at apex, pubescent to crisped-pilose, caducous. Petioles 4-6 $\times 0.8-1 \mathrm{~mm}$, grooved above either side of raised, well-defined midvein, narrowly winged, glabrescent. Leaves $7.5-11 \times 3-4.5 \mathrm{~cm}, 2-2.5$ times as long as broad, chartaceous to thinly subcoriaceous, elliptic, oblong-elliptic or ovateoblong, often asymmetric, base acute to rounded, sometimes shortly attenuate, asymmetric or not, apex cuspidate-acuminate, acumen $1.5-2 \mathrm{~cm}$ long, extreme tip acute, apiculate; lamina densely pellucidpunctate, glabrous above, usually sparsely puberulent to pubescent below, the hairs generally confined mostly to mid- and main veins, rarely pubescent to pilose over the whole undersurface. Midvein raised below, conspicuous or not. Secondary veins 5-7 pairs, strongly curved-ascending, branching from midvein at $50-70^{\circ}$, slightly raised on both surfaces. Leaf margin finely serrate throughout, the teeth close-set, often elongate. Inflorescences sessile to shortly $(c a .3 \mathrm{~mm})$ pedunculate, fasciculate/glomerate, few- to many-flowered, in foliate or defoliate axils of young twigs. Bracts numerous, $1-2 \mathrm{~mm}$ long, ovate; outermost bracts apparently subtending more than one flower, acute to obtuse at apex, pilose outside, glabrous inside; innermost bracts subtending single pedicel, membranous, fragile, glossy, ovate to rounded or truncate at apex, sparsely pilose to glabrous outside, glabrous inside. Pedicel in flower 1-2 mm long, slender, articulate near apex, pilose with white semi-spreading hairs. Bud broadly ellipsoid to urceolate. Flowers urceolate to shortcylindrical, sweetly aromatic. Calyx $2-3.5 \mathrm{~mm}$ long, slightly accrescent in fruit, whitish when fresh, fused in basal 1/3 to form a short tube, calyx lobes ('sepals') 5, broadly oblong-ovate, with apex obtuse to rounded, slightly hooded, outside adpressed pilose, sparsely so in apical half, inside glabrous, margin minutely ciliate at apex. Stamens 10; filaments $1-1.5 \mathrm{~mm}$ long, glabrous except for a few hairs at base; anther $0.2-0.3 \mathrm{~mm}$ long, glabrous, connective swollen, bifurcate, minutely apiculate, sparsely barbate. Disc cup densely pubescent on inner surface; disc lobes ca. $0.3 \mathrm{~mm}$ long, narrowly oblong, inner face pilose. Ovary $0.7-0.8 \mathrm{~mm}$ long, ovoid to broadly ellipsoid, longitudinally ridged, glabrous, ovules 2 per placenta. Style $c a .1 \mathrm{~mm}$ long, stigma capitate, barbate. Pedicel in fruit $2-$ $4 \mathrm{~mm}$ long, slender, articulate in lower $1 / 3$. Capsule 3-5.5 mm long excluding style remnant, enclosed to ca half way or more by persistent calyx, obovate to subglobose before dehiscence, 3-angled, base acute, apex rounded; pericarp ca. $0.1 \mathrm{~mm}$ thick, resinous, outside shallowly tuberculate, glabrous, inside glabrous; valves keeled, acuminate by persistent style remnant, at dehiscence erect to semi-spreading. Seed 1-4, 2.5-3 mm long, irregularly ellipsoid to compressed polygonal, testa foveolate-reticulate, orangebrown to brown; aril substantial, irregularly folded, with long, thin extensions, waxy, yellowish when fresh, drying whitish.

Habitat within Reserva Ducke: Canopy tree in low forest, by streams, on sandy soil.

Distribution within Reserva Ducke: "floresta de baixio".

General distribution: South America from Venezuela southwards to Brazil.

Material from the Reserva Ducke: Sothers 446. Additional material: BRAZIL. AMAZONAS: near mouth of Rio Embira (tributary of Rio Tarauaca), 7³0’S, 70¹5'W, 21.VI.1933(fr) Krukoff4966 (K NY); Rio Purus, Lago Prêto, $2 \mathrm{~km}$ north of Lábrea, 26.VI.1971 (fr) Prance et al. 13749 (INPA K NY US). ACRE: vicinity of Perequito, Rio Juruá-Mirim, 19.V.1971 (fr) Steward et al. P1315I (INPA K NY). GUYANA. Rupununi River, Karanambo, 345'N, 59²0’W, 1.IX.1988 (fr) Maas et al. 7154 (K U).

Species recognition. In flower: smallish oblong leaves, tertiary veins reticulate, not percurrent, hairs on leaf lower surface usually confined to venation, small flowers, ovary glabrous, style unbranched. In fruit: leaf characters as above, small fruit more than half-way enclosed by sepals. 
5.6 Casearia grandiflora Cambess. in St. Hilaire, Fl. bras. Merid. Quarto ed. 2: 168, t.126. 1830.

Casearia hypoleuca Martius, Herb. Fl. bras. 128.1837.

Shrub or small tree to $10(-15) \mathrm{m}$ tall, trunk brown. Twig tips sulcate and/or ridged, densely pale tawny-brown tomentose, older twigs and branchlets finely ridged, brown-tomentose, eventually glabrous. Lenticels obscure on younger twigs, numerous on older twigs and branchlets, barely visible without magnification. Stipules $c a$. $5 \mathrm{~mm}$ long, long-ovate, acute at apex, densely tomentose, caducous. Petioles $2-5 \times 1-1.5 \mathrm{~mm}$, shallowly rounded to flat or shallowly grooved above, not winged, densely pale-brown tomentose. Leaves $6-9.5 \times 1.8-2.7 \mathrm{~cm}, 2.7-4.5$ times as long as broad, chartaceous, oblong, more or less symmetric, base broadly acute to rounded, sometimes shortly attenuate, often asymmetric, apex cuspidate-acuminate, acumen $0.5-1 \mathrm{~cm}$ long, extreme tip obtuse, minutely apiculate; lamina practically impunctate, upper surface glabrous except for sparsely to densely tomentose midvein, lower surface densely tomentose throughout, whitish or greyish, drying pale tawny-brown. Midvein raised below, conspicuous. Secondary veins 13-15 pairs, straight-ascending for most of their length, curved-ascending towards leaf margin, branching from midvein at $40-50^{\circ}$, not well-defined on upper surface, more clearly so below. Leaf margin finely and clearly serrate throughout. Inflorescences pedunculate, glomerulate, few- to many-flowered, at anthesis in foliate or defoliate axils of young twigs; peduncle 2-4 mm long, densely tomentose. Pedicel in flower very short, $0.2-0.3 \mathrm{~mm}$ long, tomentose, articulate at base. Bracts: outer bract(s) 2.5-3 mmlong, broadly ovate, papery, 3-4-lobed, outside sericeous/ tomentose mixed, inside glabrous; innermost bracts (subtending pedicel) $3-3.5 \mathrm{~mm}$ long, at first cup-shaped, enclosing bud, later deeply 2(-3)-lobed, with the bract-lobes usually cut to base and appearing as 2-3 separate suborbicular bracts, papery, densely pale tawnybrown sericeous outside, inside glabrous, glossy, reddish-brown. Bud broad-ellipsoid to oblong, apex rounded. Flower subsessile, becoming urceolate. Calyx 7-8 $\mathrm{mm}$ long, fused in basal $1 /$ 4-1/3 to form a short tube, whitish to yellowish or greenish when fresh, calyx lobes ('sepals') 5 , erect at anthesis, oblong to oblong-obovate, with rounded, hooded apex, outside densely sericeous, indumentum pale tawny-brown, inside sparsely adpressed-puberulent. Stamens 13-15; filaments 2.5-3 mm long, glabrous; anther ca. $0.5 \mathrm{~mm}$ long, globose, sparsely barbate, connective with swollen gland, sparsely barbate. Disc cup inner surface sericeous towards base, tomentose towards rim; disc lobes 1.5-2 mm long, narrowly oblong, outer face sericeous, inner face very densely long-villous throughout, with bi-directional tufts of hair at apex. Ovary 1.5-2 mm long, narrowly ovate, tapering gradually into base of style, densely sericeous, ovules 5-8 per placenta. Style $2.5-3 \mathrm{~mm}$ long, tapering gradually to apex, densely sericeous in basal half, sparsely so towards apex; stigma capitate, densely and shortly barbate, exserted from calyx with development of fruit. Pedicel in fruit 0.2-0.5 mm. Capsule 5-8 mm long excluding persistent style remnant and stalk, shortly ( $c a .1 \mathrm{~mm}$ ) stalked, enclosed to about half way by persistent calyx, subglobose to broadly obovoid before dehiscence, longitudinally ribbed, base acute to rounded, apex obtuse to rounded; pericarp coriaceous, thin, outside usually finely and shallowly tuberculate, sparsely pilose in apical half, densely adpressedpilose at apex around persistent style base, green to orange when fresh, becoming dark red to purplish or blackish when mature, drying darkbrown to blackish, pericarp inside glossy, glabrous, with placental areas pale reddish-brown to dark cream, darker brown towards valve margins; valves at dehiscence semi-spreading. Seed 18 23 , ca. $3 \mathrm{~mm}$ long, irregularly compressedellipsoid, basal 1-2 mm usually constricted, base truncate, apex obtuse to rounded, testa foveolatereticulate, covered entirely by a thin, semitransparent membrane, turning from orange- to dark-brown; aril substantial, mostly filamentous, cream-coloured when fresh.

Sleumer(1980) described the seed of $C$. grandiflora as 3-4 in number, $c a .2 .5 \mathrm{~mm}$ in length. Seed seen for this (Reserva Ducke) account were ca. $3 \mathrm{~mm}$ long, presumably mature, but $18-23$ in 
number. Ovules counted from Reserva Ducke material numbered 5-8 per placenta.

Habitat within Reserva Ducke: Disturbed forest on sandy soil.

Distribution within Reserva Ducke: "floresta de vertente".

General distribution: South America: Panama, Colombia, Venezuela, Guianas, Brazil. Material from the Reserva Ducke: Assunção 86, 101; Sothers 939.

Additional material: BRAZIL. RORAIMA: Alto Alegre, Serra de Tepequém, 350'N, 6140'W, 23.XII.1987 (fr) Hopkins et al. 977 (INPA K MG NY). PARÁ: Serra dos Carajas, Serra Norte. ca. $6^{\circ} \mathrm{S}$, 50 $15^{\prime}$ W, 14.X.1977 (fr) Berg \& Henderson BG518 (INPA K MG NY). MARANHÃO: Road Carolina to Estreita, 2-15 km from Estreita, 9.VIII.1964 (fr) Prance \& Silva 58623 (INPA K MG NY). MATO GROSSO: Santa Terezinha, road to Santa Terezinha (MT413), $7 \mathrm{~km}$ E of jct with BR158. 10²0'S, 51 ${ }^{\circ} 13^{\prime} \mathrm{W}$, 13.X.1985 (fr) Thomas et al. 4360 (INPA K MG NY). Species recognition: In flower and fruit: combination of densely tomentose leaf undersides and subsessile flowers and fruit. C. arborea forma (see species no. 7) also has tomentose leaf undersides, but the leaves are much larger than in $C$. grandiflora, and the pedicels longer.

5.7 Casearia arborea (Rich.) Urb., Symb. Antill. 4: 421. 1910.

Tree to $25 \mathrm{~m}$ tall. Twigs and branchlets slightly sulcate, densely and minutely palebrown tomentose throughout. Lenticels numerous throughout younger twigs, partially covered by and almost the same colour as indumentum, view at $\times 10 \mathrm{mag}$. Stipules (not seen) caducous. Petioles 6-9 × 1.5-2 mm, slightly grooved and narrowly winged above, glabrous. Leaves $12-18 \times 4.5-6 \mathrm{~cm}, 2.7-3.3$ times as long as broad, thinly coriaceous, oblongelliptic, slightly asymmetric, base acute to broadly so, apex cuspidate-acuminate, acumen $0.5-1 \mathrm{~cm}$ long, extreme tip obtuse, minutely apiculate; lamina pellucid-punctate/striate, upper surface glabrous, including mid- and secondary veins, lower surface very densely and very finely greygreen tomentose, indumentum less dense on secondary veins, sparse on midvein. Midvein raised and very conspicuous below. Secondary veins 9-11 pairs, curved-ascending, branching from midvein at $50-65^{\circ}$, slightly raised above, raised and well-defined below. Leaf margin practically entire. Inflorescences pedunculate, glomerulate, several- to many-flowered, in foliate or defoliate axils of young twigs; peduncle $3-5 \times 1 \mathrm{~mm}$, densely and minutely tomentose. Bracts up to $1 \mathrm{~mm}$ long, acute to obtuse at apex, minutely and densely tomentose outside, glabrous inside. Pedicel in flower 8-10 mm long, slender, densely and minutely tomentose, articulate near base. Bud ovoid. Calyx 6-8 mm long, fused in basal 1/5-1/4 to form a short tube, calyx lobes ('sepals') 5, erect at anthesis, oblong-ovate, with apex rounded and slightly hooded, outside densely and minutely tomentose, inside glabrous. Stamens 10-11; filaments slender, 2-2.3 mm long, sparsely pilose in basal half, glabrous towards apex; anther $0.5-0.6 \mathrm{~mm}$ long, broadly ellipsoid, connective apically swollen, sparsely barbate. Disc cup inner surface glabrous below, outer surface of free rim adpressed-pilose, inner surface ferruginousvillous; disc lobes $c a .1 .5 \mathrm{~mm}$ long, narrowly oblong, slightly sigmoid, outer face adpressed pilose, inner face densely ferruginous-villous, apex densely ferruginous-villous on both surfaces. Ovary (not seen at anthesis) becoming ovoid-ellipsoid, 3-angled, glabrous in basal half, sparsely pilose in apical half, hairs mostly around style base, wall cross-section reticulate, resinous at least toward valve margins, ovules $c a .10$ per placenta. Style $c a .3 \mathrm{~mm}$ long, adpressed pilose in basal half, sparsely pilose in apical half; stigma capitate, minutely and sparsely barbate. Immature capsule globose, enclosed by calyx, ca. $0.5 \mathrm{~cm}$ long, 3-angled, dark brown, mostly glabrous, adpressed-pilose towards apex, pericarp resinous, valves keeled, style remnant persistent. Seed not seen.

Habitat within Reserva Ducke: plateau forest on clay.

Distribution within Reserva Ducke: "floresta de platô".

General distribution of $C$. arborea (Rich.) Urban sens. lat.: C. America from Guatemala southwards, South America from Venezeula southwards to Bolivia and Brazil. 
Material from the Reserva Ducke: Santos 930.

In flower Santos 930 is very similar to the more typical C. arborea (Rich.) Urban, but much larger in all its parts. Typical C. arborea is not yet known from within the Reserva Ducke but has been collected frequently from Mun. de Manaus. Santos 930 is a very good match for Rodrigues \& Coêlho 9069 (Brazil: Estrada Manaus-Itacoatiara, km 145), a collection annotated by Sleumer (1977) as ' $C$. arborea, forma luxurians'. Material approaching C. arborea forma luxurians -sensu Sleumer has also been collected from Pará: Prance et al. P.25597, 20.IX.1977 (bd) Cuiabá-Santarém Highway BR163, vicinity of Igarapé Kazuo (INPA KNY). Species recognition: See $C$. grandiflora.

Group Decandrae sensu Sleumer, Fl. Neotrop. 22: Flacourtiaceae, 289. 1980.

Fruit small to large, dry-baccate or succulent, calyx not enclosing mature fruit to half way or more, sepals or calyx-lobes reflexed at anthesis, stamens 10, equilong or slightly inequilong.

5.8 Casearia duckeana Sleumer, Fl. Neotrop. 22: Flacourtiaceae, 349. 1980.

Shrub or small tree to $12 \mathrm{~m}$ tall, trunk beigeyellow. Twig tips and young twigs sulcate/ridged, minutely spreading-puberulent, older twigs and branchlets sulcate/ridged, becoming glabrous, often whitish, minutely tuberculate by lenticels. Lenticels inconspicuous/absent on youngest twigs, on older twigs and branchlets numerous, raised, with long axis often perpendicular to long axis of stem. Stipules 3-4 mm long, linear, tapering, narrowly acute at apex, puberulent outside, early caducous. Petioles $(2-) 3-5 \times 0.7-0.8 \mathrm{~mm}$, grooved above, with margins inrolled, pubescent at first, later glabrous, often drying blackish. Leaves mostly $7-11 \times 3-4 \mathrm{~cm}$, with smaller leaves often present especially towards base of branchlets, all leaves (2-)2.5-2.8 times as long as broad, chartaceous, elliptic, symmetric, base broadly acute to rounded, apex cuspidateacuminate, acumen $0.7-2 \mathrm{~cm}$ long (smaller leaves excluded), extreme tip obtuse to acute, minutely apiculate; lamina pellucid-punctate/lineate, practically glabrous throughout on both surfaces except for a few minute hairs on midvein underside, upper surface quite glossy. Midvein raised below, not especially conspicuous. Secondary veins 3-5 pairs, curved-ascending, with basal pairs sometimes high-ascending, all pairs branching from midvein at $30-50^{\circ}$, impressed and rather weakly defined above, raised and more clearly defined below. Leaf margin shallowly serrate to subentire. Inflorescences sessile, fasciculate, in foliate or defoliate axils of young twigs, few- to manyflowered. Bracts $0.5-1.5 \mathrm{~mm}$ long, triangular to ovate, papery, outside puberulent to glabrous, inside glabrous. Pedicel in flower 8-9 mm long, filiform, articulate near base, puberulent. Buds not seen. Calyx ca. $5 \mathrm{~mm}$ long, divided almost to base, whitish-green when fresh, calyx lobes ('sepals') 5, strongly reflexed at anthesis, narrowly triangular to linear, with apex rounded and hooded, dark striated at least when dried, quite densely puberulent on both surfaces. Stamens 10, exposed by reflexion of sepals; filaments alternately inequilong, ca 3-3.5 mm long, filiform, villous in basal 1/3-1/2, otherwise glabrous; anther ca. 0.4 $\mathrm{mm}$ long, oblong-ellipsoid, glabrous, connective not swollen, glabrous. Disc cup inner surface villous; disc lobes narrowly clavate, $c a .1 .3 \mathrm{~mm}$ long, villous, densely so at apex. Ovary 1-1.2 mm long, sparsely villous, at first narrowly ovate and tapering gradually into style base, often becoming obovoid, ovules 4-5 per placenta. Style 2.5-3 mm long, rather stout in basal half, mostly villous, glabrous towards apex, apex capitate, puberulent. Pedicel in fruit 7-10 $\mathrm{mm}$ long, puberulent, articulate near base. Capsule 1.5-2 cm long, globose to broadly obovoid, usually with 3 very fine ridges marking valve margins, base acute to rounded, apex rounded, style base usually persistent; pericarp dry, lightweight, 0.5-1 mm thick, outside very densely velutinous, indumentum yellowish, drying greyish or brownish, sometimes with small dark dots and streaks just visible, pericarp inside glabrous, glossy, brown; perianth remnants at fruit base persistent or not. Seed 1-3 per capsule, immature seed up to 8 $\mathrm{mm}$ long, compressed ellipsoid-trigonal, testa rather smooth, glabrous, resinous; aril gelatinous, sweet-tasting. 
Sleumer (1980) described C. duckeana Sleum. as 3-8-flowered with 3-4 seed per capsule. Habitat within Reserva Ducke: understorey tree of plateau or disturbed forest, on clay or sandy soil.

Distribution within Reserva Ducke: "floresta de platô", "floresta de vertente".

General distribution: Brazil (Amazonas, Rondônia).

Material from the Reserva Ducke: Albuquerque 46 (not seen); Assunção 167, 168; Monteiro 50 (not seen); Nascimento 681, 774; Rodrigues 5761 (not seen), 8295 (not seen); Schultes 26121 (not seen); Sothers 404, 457, 961; Souza 145 (not seen); Vicentini 737. Species recognition: In flower: leaves 7 $11 \mathrm{~cm}$ long, produced at the same time as or after the flowers, linear to narrow triangular sepals reflexed at anthesis, stamen filaments drying pale brown to yellowish-white and disclobe indumentum drying white to yellowish. The perpendicular orientation of the lenticels to the stem may also be a useful characteristic, especially in the identification of sterile material. See also $C$. javitensis (species 2). In fruit: capsules $1.5-2 \mathrm{~cm}$ long, globose to broadly obovoid, densely greyish-velutinous.

5.9 Casearia pitumba Sleumer, Blumea 24: 118. 1978.

Casearia macrophylla Vahl, Eclog. Amer. 2: 32. 1798. nom. superfl. illeg.

Shrub or tree to $15 \mathrm{~m}$ tall, trunk beige to brown. Young twigs sulcate/ridged, glabrous, older twigs and branchlets whitish, finely pustulate-lenticellate. Stipules 3-6 mm long, linear, gradually tapering to narrowly acute tip, puberulent outside, early caducous. Petioles $5-$ $15 \times 0.8-1.4 \mathrm{~mm}$, grooved above, glabrous, petiole margins more or less inrolled, sometimes fused over petiole surface. Leaves $9.5-13.5 \times 3.5-5 \mathrm{~cm}$, 2-3 times as long as broad, chartaceous, elliptic to obovate, symmetric, base acute to rounded, attenuate or not, apex cuspidate-acuminate, acumen $0.7-2.5 \mathrm{~cm}$ long, extreme tip acute, minutely apiculate; lamina impunctate to densely pellucid-punctate/lineate, both surfaces completely glabrous including venation, often drying darkish brown. Midvein raised below, conspicuous or not.
Secondary veins (4-)5-8 pairs, curved-ascending, branching from midvein at $30-60^{\circ}$, below raised, conspicuous or not. Leaf margin shallowly serrate. Inflorescences sessile, fasciculate, fewto many-flowered, in foliate or defoliate axils of young twigs. Bracts $1.5-4 \mathrm{~mm}$ long, ovate, with apex acute to obtuse, papery, pubescent on both surfaces. Pedicel in flower $2.5-5 \mathrm{~mm}$, slender, articulate near middle or in apical half, puberulent. Bud narrowly ovoid. Calyx 6-6.5 $\mathrm{mm}$ long, fused in basal $1 / 4$ to form a short tube, whitish or greenish, calyx lobes ('sepals') erect to spreading at anthesis, narrowly oblong-ovate, with apex rounded, outside puberulent, inside glabrous in basal half, puberulent in apical half. Stamens 10; filaments alternately inequilong, $1.5-2 \mathrm{~mm}$ long, long-villous, densely so in apical half; anthers 0.5-0.6 mm long, globose-ellipsoid, glabrous to sparsely long-hispid; connective apiculate, long-hispid, the hairs erect, about as long as the anther itself and projecting conspicuously beyond the anther. Disc cup inner surface sparsely villous, glabrous towards base; disc lobes ca. $1 \mathrm{~mm}$ long, narrowly oblong, densely whitevillous, especially at apex. Ovary 1-2 mm long, at first narrowly ovate and tapering gradually into style base, glabrous or puberulent (?)*, ovules several per placenta. Style 3.5-4 mm long, rather stout and densely adpressed-villous in basal half, apical half more slender, sparsely pilose; stigma capitate, papillose (view at $\times 20$ mag.), shortly barbate. Pedicel/peduncle in fruit stout, glabrous, articulate near middle. Capsule $2.5-3 \mathrm{~cm}$ diameter, globose, apex rounded or apiculate, in dried material valve margins marked by 3 fine impressed longitudinal lines; pericarp outside irregularly and shallowly verrucose, dark green to greenish yellow or orange when fresh, usually drying darkish brown, puberulent at first, later glabrous except for hairy style-base remnant, pericarp inside glabrous, pale orangebrown. Perianth remnants persistent only as narrow rim at pedicel apex. Seed 1-few, 12-13 mm long, compressed ovoid-trigonal, testa rather smooth; aril membranous, white when fresh.

*As the young fruit of C. pitumba may be densely puberulent. 
Habitat within Reserva Ducke: forested plateau and slopes, on clay or sandy soil.

Distribution within Reserva Ducke: "floresta de platô", "floresta de vertente".

General distribution: Amazonian South America. Material from the Reserva Ducke: Coêlho 9 (not seen); Ribeiro 1622; Rodrigues 5831 (not seen), 6918 (not seen); Sothers 791; Vicentini 402.

Additional material studied: BRAZIL. AMAPA: on forested island, about $0.5 \mathrm{~km}$ south of mouth of Rio Maturá, 2`34'N, 52³2'W, 21.IX.1960 (fl) Irwin et al. 48397 (IAN K MG,NY). AMAZONAS: track from Boca do Acre air-strip to Monte Verde, north bank of Rio Purus, 21.IX.1966 (fl) Prance et al. 2465 (IAN INPAK NY). PARÁ: $1 \mathrm{~km}$. north of Rio Muirapiranga, 11.X.1965 (fl) Prance et al. 1605 (IAN K NY).

Species recognition: In flower: combination of chartaceous leaves 9.5-13.5 cm long, pedicellate flowers, relatively narrow, erect sepals and long-villous anthers, the anther hairs being almost as long as the anther itself. According to Sleumer (1980) the leaves of $C$. pitumba can eventually become subcoriaceous and such forms of the species might be confused with some forms of $C$. combaymensis. In fruit: combination of leaf form and texture and relatively large (2.5-3 cm diam.) globose and mostly glabrous capsules, these often with longitudinal lines marking valve margins in predehiscent fruit and a fairly conspicuous hairy apicula. See also C. combaymensis.

Group Aculeatae sensu Sleumer, Fl. Neotrop. 22: Flacourtiaceae, 289. 1980.

Flowers more or less cylindrical; bud obtuse truncate at base, obtuse at apex; sepals thin, erect, with apex obtuse; stamens (7-)8(-10), (sub)equal in length, anthers eglandular. Fruit glabrous or glabrescent, often tuberculate and ridged.

5.10 Casearia negrensis Eichl. in Mart. Fl. bras. 13(1): 466. 1871.

Small tree to $c a .8$ (rarely -18 ) $m$ tall, trunk brown-grey to dark reddish-brown. Twig tips sulcate, twig tips and youngest branchlets sparsely white-puberulent, becoming glabrous*. Lenticels apparently absent on younger branchlets, inconspicuous on older branchlets. Stipules 1$2.5 \mathrm{~mm}$ long, very narrowly triangular, with acute apex, hairy, eventually caducous although often at least remnants persistent for some time. Petioles $5-8 \times 1-1.3 \mathrm{~mm}$, grooved above, often narrowly winged, becoming practically glabrous. Leaves $10.5-16.5 \times 3.5-5.5 \mathrm{~cm}, 2.5-3.5$ times as long as broad, chartaceous to thinly subcoriaceous, elliptic to oblong-elliptic, more or less symmetric, base acute to broadly so, rarely obtuse or rounded, apex cuspidate-acuminate, acumen 1-2 cm long, extreme tip acute, minutely apiculate; lamina densely but rather obscurely pellucid-punctate, also with 1-few larger, dark-bordered, pale-orangebrown irregular pellucid punctations/striations within most aereolae, upper surface glabrous, usually somewhat glossy, lower surface with just a few short hairs scattered on mid- and secondary veins. Midvein raised below, conspicuous. Secondary veins $8-10$ pairs, curved-ascending, branching from midvein at $60-80^{\circ}$, raised on both surfaces, especially conspicuous below, tertiary veins percurrent i.e. ladder-like, close set, more or less straight, parallel to one another, all running at $c a .90^{\circ}$ to midvein, often raised and conspicuous on both surfaces. Leaf margin distantly and minutely toothed to almost entire. Inflorescence sessile, fasciculate, few- to several-flowered, generally in foliate axils of young twigs. Bracts numerous, 1-2 mm long, ovate, acute to obtuse at apex, membranous to papery, outside glabrous or with a longitudinal band of straight, adpressed hairs, inside more or less glabrous, margins glabrous or ciliate. Pedicels mostly 3-5(-7) mm long, sparsely pubescent. Bud ellipsoid to oblongellipsoid, obtuse to rounded at base and apex. Calyx 5-5.5 mm long, fused in basal $0.5 \mathrm{~mm}$ to form a very short tube, greenish white when fresh, calyx lobes ('sepals') 5, erect to spreading at anthesis, sometimes reflexed in fruit, oblongelliptic, with apex rounded, outside glabrous, with dark streaks and dots, inside sparsely shortsericeous. Stamens $6^{*}$, filaments equilong, $c a$. $2 \mathrm{~mm}$ long, glabrous or sparsely hairy in basal half; anther $0.8-1 \mathrm{~mm}$ long, long-ellipsoid to

\footnotetext{
*In material of C. negrensis from outside of the Reserve (e.g. Amazonas, Daly et al. 4042, 5415) the terminal bud is quite densely ferruginous adpressed hairy, and the twig tips ferruginous crisped-pubescent. According to Sleumer (1980) C. negrensis usually has 8 stamens.
} 
ovoid-triangular, connective linear to narrowly lanceolate, not swollen, glabrous. Disc cup glabrous inside; disc lobes 1-1.2 mm long, broadly oblong, with apex rounded to truncate, half as long as filaments, villous on inner face and at apex. Ovary 1-1.5 mm long, ovoid to globose, tapering gradually into base of style, mostly glabrous, sericeous at apex. Style $1.5 \mathrm{~mm}$ long, basal half rather stout, sericeous at base, tapering gradually in glabrous apical part; stigma capitate, puberulent. Pedicel in fruit 4-5 mm long, articulate at base, with sparse, short, close-adpressed hairs. Capsule 2-3 cm long, broadly ellipsoid, 3-6-angled before dehiscence, purplish-green, violet, dark red or blackish when fresh, dark brown when dried, tardily dehiscent; pericarp fleshy, mostly $0.5-$ $1 \mathrm{~mm}$ thick, outside with conspicuous veins and wrinkles ( $\times 10$ mag.), glabrous except for a few short, straight close-adpressed hairs at apex, pericarp inside glabrous, pale yellowish-straw coloured, glossy, with fine horizontal striations. Perianth remnants persistent at fruit base. Seed 4-several, compressed ellipsoid-polygonal, finely striated, orange-brown; aril membranous.

Habitat within Reserva Ducke: disturbed forest, on clay or sandy soil.

Distribution within Reserva Ducke: "floresta de platô", "floresta de vertente".

General distribution: Amazonian SouthAmerica. Material from the Reserva Ducke: Assunção 266; Sothers 1064; Souza 284.

Additional material: BRAZIL.AMAPÁ: Macapá, Rio Falsino, approx. $10 \mathrm{~km}$ upstream of confluence with Rio Araguari, west bank, 050'S, 51²4'W, 13.XII.1984 (fr) Daly et al.3862 (INPA K MG NY); Macapá, Serra do Navio, $6 \mathrm{~km}$ NNW on Serra do Navio-Agua Branca Road, $1^{\circ} 03^{\prime}$ N, 52 $2^{\circ} 04$ 'W, 3.I.1985 (fr) Daly et al. 4042 (INPA K NY); W. bank of Rio Falsino, approx. $10 \mathrm{~km}$ upstream from confluence with Rio Araguari, $01^{\circ} 00^{\prime} \mathrm{N}$, 514’'W, 28.IX.1987 (fr) Pruski et al. 3291 (INPAKMG NY). AMAZONAS: São Gabriel da Cahoeira, Morro dos Seis Lagos, 0¹8’N, 66²'’W, 250-450m., 14.X.1987 (bd) Daly et al.5415 (INPA KNY). RONDÔNIA: $15 \mathrm{~km}$ $\mathrm{N}$ of Ariquemes on hwy. BR 364 and $1 \mathrm{~km}$ E on "Linea 75”. 947’'S, 6305’W, 13.III.1987 (fr) Nee 34360(KNY).

Species recognition: In flower: leaves practically glabrous above and below, usually subcoriaceous, percurrent tertiary venation, sepals glabrous to laxly hairy outside and spreading (but not reflexed) at anthesis, style simple, stamens
(6-)8, fertile axils non-resinous. In fruit: percurrent tertiary leaf venation, capsule fleshy, veined, broadly elliptic, $2-3 \mathrm{~cm}$ long.

5.11 Casearia resinifera Spruce ex Eichl. in Mart., Fl. bras 13(1): 446. 1871.

Treelet to $\mathrm{ca}$. $6 \mathrm{~m}$ tall, trunk pale chestnutbrown. Terminal bud usually resin-covered. Twigs and branchlets sulcate/ridged, glabrous, dark reddish-brown. Lenticels apparently absent/ inconspicuous on younger twigs, scattered on older twigs and branchlets. Stipules 2-2.5 mm long, broadly ovate, subcrassate, papillose, resin-covered, base rounded to shallowly subcordate, broadly obtuse to rounded at apex, caducous. Petioles 7$10 \times 1.5-2.2 \mathrm{~mm}$, glabrous, upper surface strongly grooved, margins inrolled. Leaves 14-28 × (4.5-) 6-9 cm, 3-3.7 times as long as broad, chartaceous, oblong to long-oblong, less often long-elliptic, symmetric, base acute to obtuse, apex cuspidateacuminate, acumen $2-2.5 \mathrm{~cm}$ long, extreme tip obtuse, minutely apiculate or not; lamina sparsely to densely pellucid-punctate/lineate, both surfaces glabrous throughout including venation, drying darkish brown. Midvein raised below, conspicuous. Secondary veins 10-14 (-17) pairs, curved-ascending, branching from midvein at (60-) $70-80^{\circ}$, raised on both surfaces. Leaf margin coarsely and shallowly serrate/dentate to almost entire. Inflorescences sessile, fasciculate, fewto many-flowered, resinous, in foliate (usually) axils of young twigs or older branchlets. Bracts numerous, small, suborbicular, irregularly and deeply lobed, glabrous, bract-cushion usually resin-coated. Pedicel in flower 0.5-1 mm long. Bud cylindrical to narrowly obovate, apex rounded. Flower with a slight, disagreeable smell. Calyx 5-9 mm long, fused in basal 2/3 to form a tube, pale yellowish or light pinkish when fresh, calyx lobes ('sepals') 4, erect at anthesis, oblong, with apex obtuse to rounded and hooded, glabrous on both surfaces. Stamens 8; filaments inequilong, 0.5-1 mm long, glabrous; anther 0.8$1 \mathrm{~mm}$ long, triangular-ovoid, glabrous, connective not swollen, glabrous. Disc cup glabrous inside; disc lobes small, ca. $0.5 \mathrm{~mm}$ long, linear, densely pubescent. Ovary ca. $2 \mathrm{~mm}$ long, cylindrical, glabrous. Style very short, ca. $1 \mathrm{~mm}$ long, glabrous, capitate. Pedicel in fruit 1-2 mm long, stout, 
glabrous. Fruit baccate, or possibly a tardily dehiscent capsule, 2.5-3 cm diameter, globose, rounded at base and apex, purplish, drying brown to dark brown, pericarp leathery and irregularly wrinkled when dried, $1-1.5 \mathrm{~mm}$ thick, laminate in cross-section, outside finely and shallowly tuberculate, glabrous, resinous; inside glabrous. Perianth remnants persistent. Seed numerous, ovoid-angular, smooth, 1-1.6 cm long (not seen, described from Sleumer 1980).

Habitat within Reserva Ducke: plateau or campinarana forest, clay or sandy soil.

Distribution within Reserva Ducke: "floresta de campinarana", "floresta de platô". General distribution: Amazonian SouthAmerica. Material from the Reserva Ducke: Ferreira 94/ 57; Pruski 3236; Ribeiro 1152; Rodrigues s.n. (Brazil, Amazonas, Manaus-Reserva Florestal Ducke. 26.8.57); Sothers 278, 729, 945, 1031, 1051. Additional material: BRAZIL. AMAZONAS: São Paulo de Olivença, 16.X1942 (fl) Ducke 1106 (IAN K). Species recognition: Usually Casearia resinifera can be recognised in flower or fruit by the resin covering its twig tips and fertile axils. Also, in flower: leaves oblong to long ellipticoblong, 14-28 × (4.5-)6-9 cm, secondary veins 10-14(-17) pairs, curved branching from midvein at $(60-) 70-80^{\circ}$, calyx $5-9 \mathrm{~mm}$, narrowly tubular in basal 2/3, disc lobes ca. $0.5 \mathrm{~mm}$ long. In fruit: combination of leaf-shape and size and relatively large, globose, glabrous capsules. See also note at end of key to genera in flower.

\section{Neoptychocarpus}

Neoptychocarpus Buchheim, Taxon 8: 76. 1959.

6.1 Neoptychocarpus apodanthus (Kuhlm.) Buchheim, Taxon 8: 76. 1959.

Ptychocarpus apodanthus Kuhlm., Arch. Jard. Bot. Rio de Janeiro 4: 358, t. 31, f. a-g. 1925.

Shrub or small tree $1.5-5(-8) \mathrm{m}$ tall, dioecious or possibly monoecious.* Terminal bud and twig tips glabrous. Stipules to $c a .1 \mathrm{~mm}$ long, subovate, scarious, lacerate. Petioles 3-6(-10) $\mathrm{mm}$ long. Leaves (6-) 10-23 × (2-)2.5-7 cm, chartaceous to subcoriaceous, mostly narrow-oblong

*Ducke 34672 \& 34673 (K RB) seems to have both staminate and carpellate flowers. or narrow-elliptic, densely pellucid-punctate; base acute to rounded, apex acuminate, cuspidate or caudate for 1-1.5 cm; lamina glabrous on both surfaces including venation. Midvein raised below, sometimes very conspicuous, often drying dark reddish brown. Secondary veins (15-)2026 pairs, branching from the midvein at $\mathrm{ca} .90^{\circ}$, forking widely near leaf margin into two branches of more or less equal strength, one branch turning towards the leaf apex, the other towards leaf base, the branches of adjacent secondary veins forming loops at leaf margin. Leaf margin minutely serrate to entire. Inflorescences of 1-3 flowers in bracteate fascicles in foliate or defoliate axils. Bracts and bracteoles $2-5 \mathrm{~mm}$ long, broadly ovate to suborbicular, obtuse to rounded at apex, firm, glabrous on both surfaces. Pedicels practically 0 . Flowers unisexual, urceolate (Sleumer 1980), white (drying brown). Calyx $8-$ $11 \mathrm{~mm}$ long, fused in basal 2/3-3/4 to form narrow tube, glabrous, calyx lobes ('sepals') 4, 2-3 mm long, broadly ovate. Staminate flowers: stamens 8, perigynous, inserted on cup-like disc adnate to calyx tube, 4 longer filaments opposite calyx lobes, ca. $1 \mathrm{~mm}$ long, planate, broadly triangular with 1 pair narrowly triangular to falcate lateral lobes (or at least small teeth) at about halfway, fleshy, glabrous, 4 shorter filaments alternating with calyx lobes, almost absent; anthers $1-1.5 \mathrm{~mm}$ long, narrowly elliptic, longitudinally dehiscent, glabrous; ovary rudimentary. Carpellate flowers: ovary ovoid-pyramidate, rugose to almost lamellate, with 3-4 multi-ovulate placentas, glabrous; styles 3-4, short, 1-1.5 mm long, connate in lower half, stigmas $c a .0 .5 \mathrm{~mm}$ long, flattened, branched, papillate. Capsule sessile, 2-2.5 cm diam., globose, pericarp coriaceous, covered with smallish flattish triangular emergences or thin low irregular lamellae $c a$. $2 \mathrm{~mm}$ high, shiny red when fresh, drying orangebrown to darker brown, tardily dehiscent by 3-4 valves, before dehiscence the valve margins marked by fine, smooth, raised ridges. Seed numerous, $c a .9 \mathrm{~mm}$ long, sericeous. Habitat: understorey of forest on terra firma at low altitudes, locally dominant.

General distribution: Guianas and Amazonian Brazil. 


\section{LIST OF ACCEPTED NAMES AND SYNONYMS}

Alphabetical by taxon; accepted names in bold; taxon numbers not equivalent to numbering in main text.

\begin{tabular}{|c|c|}
\hline 1. Carpotroche crispidentata Ducke & 18. Casearia singularis Eichl. $=6$ \\
\hline 2. Casearia arborea (Rich.) Urb. & 19. Casearia sylvestris Swartz var. sylvestris \\
\hline 3. Casearia belizensis Standl. $=23$ & 20. Casearia subopaca Triana \& Planch. $=6$ \\
\hline 4. Casearia bicolor Urb. $=23$ & 21. Laetia casearioides Sagot $e x$ Benth. $=23$ \\
\hline 5. Casearia blanchetiana Miq. $=16$ & 22. Laetia cupulata Spruce ex Benth. \\
\hline 6. Casearia combaymensis Tul. & 23. Laetia procera (Poepp.) Eichl. \\
\hline 7. Casearia duckeana Sleumer & 24. Lindackeria paludosa (Benth.) Gilg \\
\hline 8. Casearia grandiflora Cambess. & 25. Neoptychocarpus apodanthus (Kuhlm.) \\
\hline 9. Casearia hypoleuca Mart. $=8$ & Buchheim \\
\hline 10. Casearia javitensis Kunth & 26. Ptychocarpus apodanthus Kuhlm. $=25$ \\
\hline 11. Casearia macrophylla Vahl $=15$ & 27. Ryania pyrifera (Rich.) Uitten \& Sleumer \\
\hline 12. Casearia manausensis Sleumer & 28. Ryania pyrifera var. subuliflora Sandw. $=30$ \\
\hline 13. Casearia negrensis Eichl. & 29. Ryania sagotiana Eichl. $=28$ \\
\hline 14. Casearia petraea Benth. $=16$ & 30. Ryania speciosa Vahl var. subuliflora (Sandw.) \\
\hline 15. Casearia pitumba Sleumer & Monach. \\
\hline $\begin{array}{l}\text { 16. Casearia ulmifolia Vahl ex Vent. } \\
\text { 17. Casearia resinifera Spruce ex Eichl. }\end{array}$ & 31. Xylosma tessmannii Sleumer \\
\hline
\end{tabular}

\section{Lindackeria}

Lindackeria C. Presl, Reliq. Haenk. 2(2): 89. 1835.

7.1 Lindackeria paludosa (Benth.) Gilg in Engl. \& Prantl., Nat. Planzenfam. Ed 2, 21:404. 1925.

Shrub or small tree, $4-6(-15) \mathrm{m}$ tall. Terminal bud and twig tips glandular puberulent. Stipules linear, $1-1.5 \mathrm{~mm}$ long, caducous. Petioles $2-8 \mathrm{~cm}$ long. Leaves $12-20(-27) \times$ $4-8(-11) \mathrm{cm}$, chartaceous to subcoriaceous, broadly elliptic to elliptic-oblong, less often ovate, minutely glandular-punctate; base acute to rounded; apex acuminate, caudate or subfalcate for 1-2 cm; lamina glabrous above, adpressed puberulent beneath including venation. Main vein raised and conspicuous below. Secondary veins 5-6 (-7) pairs, branching from midvein at 45$60^{\circ}$. Inflorescences racemose, axillary, raches slender, $1-3 \mathrm{~cm}$ long, flowers few to many (rarely -25) per raches in fascicles of 1-3. Flowers bisexual or with staminate flowers in the same inflorescence. Pedicels 3-5 mm long in staminate flowers, $6-10 \mathrm{~mm}$ long in bisexual flowers, sparsely puberulent. Sepals 3, 5-6 mm long, obovate-oblong, longitudinally parallelveined, sparsely puberulent to subglabrous outside. Petals 6(-8), oblong, 6-8 $\mathrm{mm} \times c a .2$ $\mathrm{mm}$, whitish or cream venation and indumentum as sepals. Disc absent. Stamens (20-)30-40, hypogynous, filaments $1.5-2(-3) \mathrm{mm}$ long, slender, sparsely puberulent in lower half; anthers 2-3 mm long, linear, longitudinally dehiscent, sparsely puberulent. Ovary ovoid, covered with numerous thickish glandular-pubescent globose to finger-like emergences $c a$. $1 \mathrm{~mm}$ long, placentas 3-4, multi-ovulate. Capsule 1.5-2.5 cm diam., subglobular, tardily dehiscent by $3-4$ valves, brownish-yellow when fresh, drying brownish-orange, pericarp covered with smallish conical more or less apiculate protuberances or short conical prickles $c a$. 1-2 mm long, perianth early caducous, style persistent for some time. Seed 2-3, ca. $8 \mathrm{~mm}$ diam., arillate.

Habitat: in primary forest substage or border on hilly terra firma, also in secondary growth. General distribution: widely distributed throughout the Amazon basin.

\section{ACKNOWLEDGEMENTS}

I would like to thank the following for their invaluable help: Instituto Nacional de Pesquisas da Amazônia (for the loan of material), Mike Hopkins and the Ducke Project team, Cynthia Sothers, Susannah Stuart-Smith, Gill Challen, Don Kirkup and Nicky Biggs (Adobe ${ }^{\circledR}$ Photoshop ${ }^{\circledR}$, file management). 